ARTICLE

Received 13 May 2015 | Accepted 25 Jun 2015 | Published 11 Aug 2015

DOI: $10.1038 /$ ncomms8924 OPEN

\title{
Whole-central nervous system functional imaging in larval Drosophila
}

William C. Lemon ${ }^{1, \star}$, Stefan R. Pulver ${ }^{1, \star}$, Burkhard Höckendorf ${ }^{1}$, Katie McDole ${ }^{1}$, Kristin Branson ${ }^{1}$, Jeremy Freeman ${ }^{1}$ \& Philipp J. Keller ${ }^{1}$

Understanding how the brain works in tight concert with the rest of the central nervous system (CNS) hinges upon knowledge of coordinated activity patterns across the whole CNS. We present a method for measuring activity in an entire, non-transparent CNS with high spatiotemporal resolution. We combine a light-sheet microscope capable of simultaneous multi-view imaging at volumetric speeds 25 -fold faster than the state-of-the-art, a whole-CNS imaging assay for the isolated Drosophila larval CNS and a computational framework for analysing multi-view, whole-CNS calcium imaging data. We image both brain and ventral nerve cord, covering the entire CNS at 2 or $5 \mathrm{~Hz}$ with two- or one-photon excitation, respectively. By mapping network activity during fictive behaviours and quantitatively comparing high-resolution whole-CNS activity maps across individuals, we predict functional connections between CNS regions and reveal neurons in the brain that identify type and temporal state of motor programs executed in the ventral nerve cord.

\footnotetext{
${ }^{1}$ Howard Hughes Medical Institute, Janelia Research Campus, 19700 Helix Drive, Ashburn, Virginia 20147, USA. * These authors contributed equally to this work. Correspondence and requests for materials should be addressed to P.J.K. (email: kellerp@janelia.hhmi.org) or to S.R.P. (email: pulvers@janelia.hhmi.org).
} 
$\mathrm{n}$ recent years, advances in neurogenetics and live imaging technology have reshaped the way neurobiologists approach long-standing questions. A new generation of genetically encoded calcium indicators ${ }^{1,2}$ in conjunction with sophisticated systems for targeted expression of transgenes allow for non-invasive recording of activity across large populations of neurons $s^{3-5}$. In parallel, development of advanced imaging technology such as light-sheet microscopy and light-field microscopy have facilitated high-speed, high-resolution threedimensional (3D) imaging of large neural tissue volumes ${ }^{6-13}$. Together, these technologies bring into reach the possibility of recording from every part of a nervous system during behaviours. Not only would the ability to simultaneously record activity everywhere within an entire central nervous system (CNS) enable measurements of large-scale network dynamics and provide a way to discover and map functional connections between remote CNS regions ${ }^{14}$; whole-CNS functional imaging, that is, simultaneous imaging of both brain and nerve cord, would also afford researchers with opportunities to comprehensively record from motor circuitry while simultaneously imaging activity across the brain. Such a method would thus make it possible to systematically study how brain and nerve cord interact to generate behaviour.

Owing to fundamental technical challenges ${ }^{15}$, however, it has so far not been possible to perform whole-CNS functional imaging in a nervous system larger than that of Caenorhabditis elegans $^{11}$. Even in fairly transparent specimens, such as the larval zebrafish, large-scale functional imaging has been limited to the brain ${ }^{7,8,16}$. There are three major challenges to overcome these limitations: development of imaging technology that tracks activity at physiologically relevant timescales while also maximizing physical coverage and spatial resolution throughout the CNS; development of preparations that allow imaging of neural activity in all parts of the CNS during behaviours; and development of computational methods for interpreting complex patterns of activity at the whole-CNS level and for comparing results across individuals.

The larval fruit fly Drosophila melanogaster offers an attractive opportunity for realizing functional imaging of an entire, complex CNS. The larval fruit fly is a genetically tractable model with a sophisticated toolkit for controlling transgene expression ${ }^{5,17-19}$ and a CNS that retains the ability to produce locomotor patterns in isolation from sensory input ${ }^{20,21}$. However, similar to most model organisms used for functional imaging studies, Drosophila larvae also have a fairly opaque nervous system that represents a fundamental problem for comprehensive imaging with light microscopy.

The objective of this paper is to address the three central challenges outlined above and introduce a complete methodological framework-from sample preparation and functional imaging to image analysis-for discovering and analysing functional relationships throughout the entire, non-transparent CNS of larval Drosophila. Our methodological framework combines three novel components, each of which is designed to overcome one of the three limitations.

First, we present a light-sheet microscope that enables highspeed multi-view functional imaging with one- or two-photon excitation. Multi-view imaging is essential for optimizing physical coverage of non-transparent specimens ${ }^{22-25}$, but state-of-the-art technology $22,23,26$ is by one to two orders of magnitude too slow to resolve the sub-second timescales relevant for functional imaging with calcium indicators. In order to advance imaging performance beyond this critical threshold, we developed a new multi-view imaging technique, termed high-speed simultaneous multi-view (hs-SiMView) microscopy, that improves temporal resolution in Drosophila-sized samples by 25 -fold.
Second, we integrated these capabilities with a sample preparation strategy and imaging assay for the isolated larval CNS, and demonstrate, for the first time, to our knowledge, functional imaging of neural activity in the entire, nontransparent CNS of a higher invertebrate. We used this assay to image coordinated motor activity patterns throughout the CNS for up to an hour, acquiring 20,000 volumes per sample at a sustained volumetric imaging speed of $5 \mathrm{~Hz}$.

Third, we developed a computational framework for processing and analysing multi-view calcium imaging data produced by hs-SiMView microscopy at a rate of several terabytes per experiment. This framework facilitates mapping functional relationships across the CNS during fictive behaviours and allows quantitative comparison of high-resolution, 3D neural activity maps across multiple nervous systems.

We use our combined system to characterize large-scale patterns of brain activity associated with different locomotor states in the nerve cord, predict functional connections between disparate CNS regions and identify neurons with activity patterns that are representative of the types and temporal states of different motor programs.

\section{Results}

Light-sheet microscopy for whole-CNS functional imaging. Light-sheet-based functional imaging has proven to be highly effective for observing activity in large populations of neurons. Existing microscopes offer single-view imaging capability suitable for monitoring neural activity in the brain of transparent zebrafish larvae $e^{7-9}$ or in surface regions of mammalian neural tissues $6,10,13$. In a non-transparent, highly light-scattering CNS, such as that of D. melanogaster, this approach can only partially penetrate the specimen for fluorescence excitation and detection. Single-view imaging thus gives access to only a small fraction-approximately one quarter-of the Drosophila CNS, and coverage of the entire CNS critically requires multi-view imaging capability with at least four complementary views (Supplementary Fig. 1). However, state-of-the-art microscopes with such multi-view imaging capability, such as SiMView light-sheet microscopy, were designed for imaging applications in developmental biology $22,23,26$, and are by at least one to two orders of magnitude too slow for system-level functional imaging.

Here we advance SiMView microscopy and present a light-sheet microscope capable of simultaneous multi-view imaging at the speed required for calcium imaging (Fig. 1). This hs-SiMView microscope offers functional imaging with both oneand two-photon excitation, and is the result of four advances spanning microscope design, microscope control and real-time data handling. These concepts are briefly summarized in the following and described in detail in the Methods section ('Light-sheet microscope for multi-view functional imaging' and Supplementary Fig. 2).

First, we redesigned the SiMView microscope (Fig. 1b, Supplementary Fig. 2 and Supplementary Data 1). Conventional SiMView light-sheet imaging relies on maintaining a stationary optical arrangement and moving the specimen through the light sheets with motorized samples, which fundamentally limits volumetric imaging speed ${ }^{22}$. In hs-SiMView, light sheets and detection focal planes are moved in synchrony relative to the stationary specimen location for 3D optical sectioning, that is, objective positions are continuously adjusted via piezo positioners ${ }^{7}$, light sheets are rapidly translated with galvanometer scanners and the specimen itself is kept stationary (Fig. 1c). This concept is crucial for improving speed fundamentally beyond the limit imposed by the conventional SiMView design but it also 
a

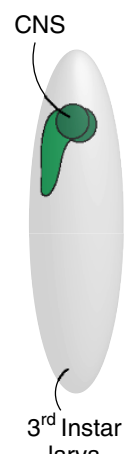

(1)

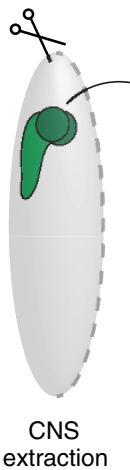

(2)

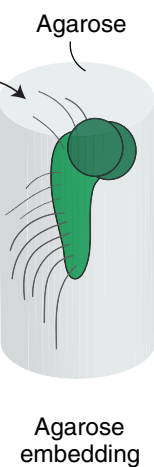

(3)

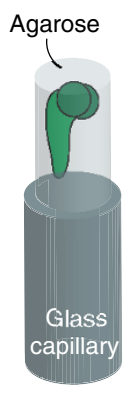

Microscope specimen holder

(4)

C

Spatiotemporally matched multi-piezo and laser scanning

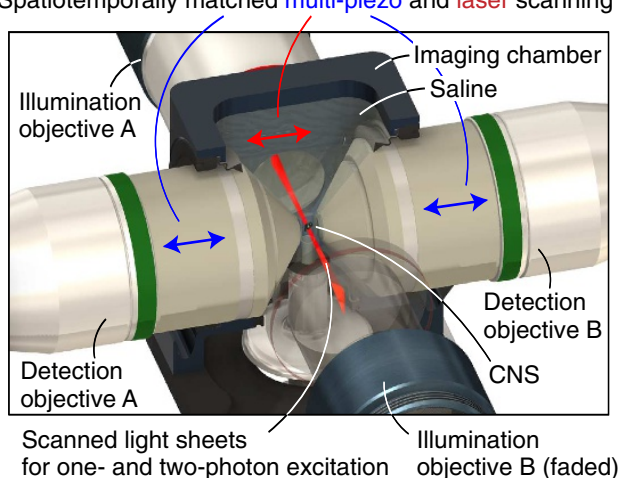

b Custom-fine adjustment I Ilumination objectives

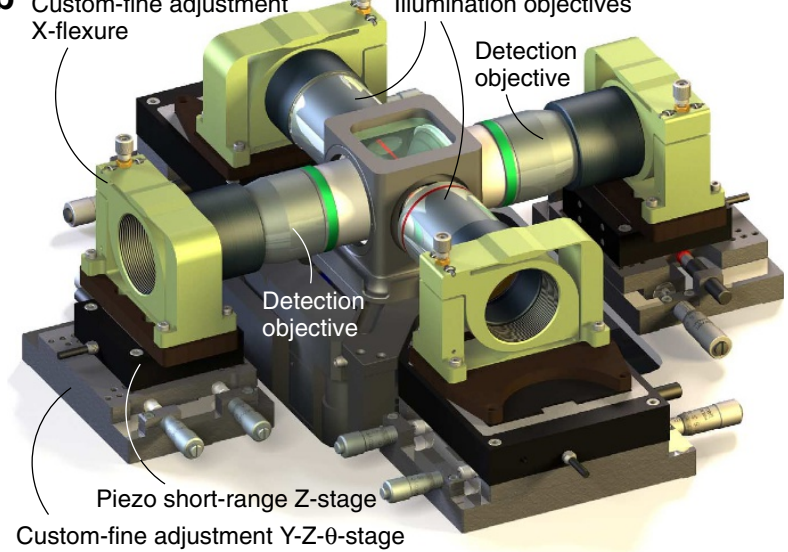

d

Multi-view functional 4D image data

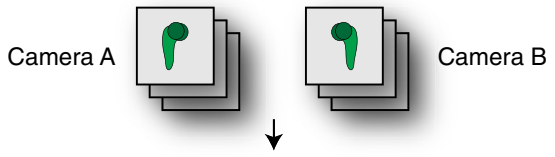

Multi-view image fusion, drift correction and $\Delta F / F$ calculation

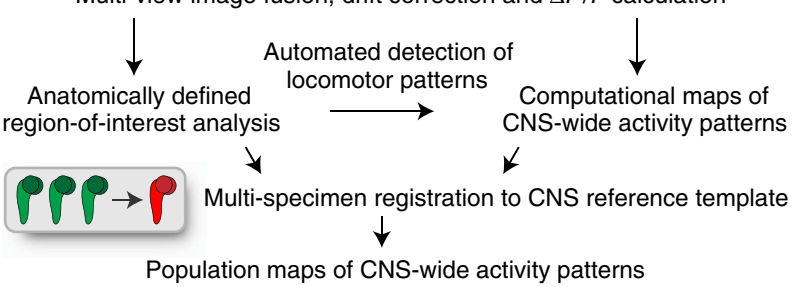

Figure 1 | Light-sheet microscopy and computational tools for whole-CNS functional imaging. (a) For optimal optical access, the CNS of a Drosophila third (3rd) instar larva is extracted by surgery and embedded in a soft, transparent agarose cylinder supported by a glass capillary for mounting in the hs-SiMView light-sheet microscope. (b) The CNS explant is then transferred to the microscope's specimen chamber filled with physiological saline. The illustration shows the hs-SiMView microscope core for SiMView functional imaging, including the central specimen chamber, two illumination objectives for bi-directional fluorescence excitation with scanned laser light sheets and two opposing detection objectives mounted on high-speed piezo stages. The 3D volumes covered by the two piezo-operated detection objectives are matched with a precision of a few micrometres using custom $Y$-Z- $\theta$ fine adjustment stages and objective $X$-flexures. (c) In conventional multi-view light-sheet microscopy, the specimen is physically moved through the light sheet for volumetric imaging, which fundamentally constrains imaging speed. In contrast, high-speed multi-view volumetric imaging in hs-SiMView is achieved by keeping the specimen stationary and instead co-translating the focal planes of the two opposing detection systems using piezos.

Simultaneously, the thin specimen volume at the location of the focal planes is illuminated bi-directionally with light sheets translated by spatiotemporally matched laser scanning. (d) Using a custom processing pipeline for hs-SiMView functional imaging data, the geometry of the specimen is automatically determined from multi-view image data, image foreground is stored using lossless compression in a custom block-based file format, multiple camera views are spatially registered, multi-view image data are fused, specimen drift is compensated locally and globally across the time-lapse experiment, and a $\Delta F / F$ representation of the hs-SiMView time-lapse data set is computed. Using a custom data analysis pipeline, locomotor activity patterns are automatically detected and classified, and high-resolution computational maps of CNS-wide activity timing are constructed for multiple fictive behaviours. In order to quantitatively compare CNS-wide activity maps across multiple nervous systems, a CNS template is constructed from all data sets and subsequently used to transform all image data into a common reference coordinate system using nonlinear spatial registration.

introduces several challenges for multi-view imaging. High-speed piezo positioning requires the use of high-stiffness piezos with short travel range on the order of no more than $100-250 \mu \mathrm{m}$. Thus, positions and orientations of the 3D fields-of-view associated with each of the four optical arms must be precisely matched to maximize the overall size of the specimen volume accessible by all arms. At the same time, the translational and rotational degrees of freedom required for this purpose must be implemented through a design that minimizes the mechanical footprint and added load on the piezo positioners. These requirements rule out commercially available multi-axis positioning technology, as the relatively large mass of such components would significantly impact piezo performance and thereby slow down the imaging process. We therefore developed custom four-axis stage and flexure systems for the key mechanical positioning tasks in the microscope (Fig. 1b and Supplementary
Fig. 2), ensuring robust $3 \mathrm{D}$ volume matching with micrometre precision without compromising piezo performance. To enable the reproduction of the imaging technology described here, we provide detailed technical drawings of these critical custom parts of the hs-SiMView microscope (Supplementary Data 1).

Second, we developed a control/acquisition software framework for high-speed piezo-based multi-view imaging. This framework also eliminates previous limitations in raw image acquisition speed $^{7,22}$, thereby enabling acquisition of up to 10 terabytes of image data at a sustained rate of $1 \mathrm{~GB}$ per second.

Third, we developed a strategy for multi-view microscope alignment that improves spatial overlap of the four focal planes and light sheets throughout the specimen volume. In order to reduce degradation of image quality resulting from spatially varying shifts between focal planes and light sheets (resulting from non-planar optical interfaces along the light path and 
heterogeneous optical sample properties), hs-SiMView adjusts relative positions of light sheets and detection focal planes, respectively, across the specimen volume on the basis of manual measurements at the onset of the imaging experiment.

Fourth, we developed a multi-view functional imaging assay using two-photon excitation that complements the high-speed imaging mode using one-photon excitation and provides an improved signal-to-background ratio at a reduced imaging speed (180 frames per second with two-photon hs-SiMView versus 370 frames per second with one-photon hs-SiMView).

As a result of the improvements outlined above, hs-SiMView is capable of multi-view functional imaging of a third instar larval CNS-sized volume of $500 \times 200 \times 200 \mu \mathrm{m}^{3}$ at $5 \mathrm{~Hz}$ with one-photon excitation (Supplementary Movies 1 and 2) and at $2 \mathrm{~Hz}$ with two-photon excitation (Supplementary Movies 3-5), that is, 25 -fold faster than volumetric imaging of similar-sized specimens in previous multi-view light-sheet microscopes ${ }^{22,23}$ (see Methods section and Supplementary Methods).

Estimating spatial resolution across the CNS of larval Drosophila. We systematically estimated spatial resolution as a function of location within CNS preparations (see 'Spatial resolution analysis' in Supplementary Methods section). This analysis takes advantage of fluorescent markers expressed in the nuclei of all cells and provides detailed 3D maps of spatial resolution for various developmental stages (Supplementary Figs 3-6 and Supplementary Movies 6-8). These maps show that our light-sheet-based imaging method achieves cellular or even subcellular resolution throughout non-neuropil regions of the isolated first and second instar larval CNS (Supplementary Figs 4 and 5, and Supplementary Movies 6 and 7), and for a large fraction of non-neuropil regions of the isolated third instar larval CNS (Supplementary Fig. 6 and Supplementary Movie 8). We found that, at a regional level, average resolution is highest in the ventral nerve cord (VNC), whereas in the brain (brain lobes and suboesophageal ganglion, SOG) relative resolution is reduced as a result of the longer average illumination and detection path lengths: in first and second instar stages, lateral and axial resolution are on average 22 and $8 \%$, respectively, better in VNC versus brain; and in the third instar stage, lateral and axial resolution are on average 14 and 44\%, respectively, better in VNC versus brain. Our estimates of average lateral/axial resolution throughout non-neuropil regions are $1.0 / 2.5 \mu \mathrm{m}$ for the first instar stage, $1.1 / 3.2 \mu \mathrm{m}$ for the second instar stage and $1.6 / 5.9 \mu \mathrm{m}$ for the third instar stage (for detailed statistics, please see 'Spatial resolution analysis' in Supplementary Methods section).

Functional imaging of the isolated Drosophila CNS. We expressed the genetically encoded calcium indicator GCaMP6s panneuronally in third instar larvae ${ }^{1}$ and imaged fluorescence changes associated with neural activity in the isolated CNS using hs-SiMView microscopy with one-photon excitation (Fig. 2 and Supplementary Movies 1 and 2) or two-photon excitation (Supplementary Movies 3-5), respectively. Before imaging, CNS explants were embedded in low-melting-point agarose and immersed in physiological saline (Fig. 1a-c). These embedded preparations produced a range of segmentally coordinated activity patterns including waves of anterior progressing and posterior progressing activity in the VNC for up to an hour (Figs 2 and 3, and Supplementary Movies 1-5). These waves represent fictive forward and backward crawling motor patterns, respectively ${ }^{20,21,27}$.

We compared frequency and duration of forward and backward waves in embedded preparations to those in agarose-free controls (Fig. $3 \mathrm{c}-\mathrm{h}$ ). In embedded preparations, wave durations were $9.3 \pm 3.7$ and $4.7 \pm 1.1 \mathrm{~s}$, and wave frequencies were $9.5 \pm 4.1$ and $15.5 \pm 9.0 \mathrm{mHz}$ for forward and backward waves, respectively (mean \pm s.d., $n=6$ preparations). In agarose-free preparations, wave durations were $10.0 \pm 2.7$ and $5.0 \pm 0.3 \mathrm{~s}$, and wave frequencies were $10.3 \pm 4.6$ and $12.4 \pm 5.9 \mathrm{mHz}$ for forward and backward waves, respectively (mean \pm s.d., $n=5$ preparations). Overall, no significant differences in wave frequency or duration were observed between embedded and agarose-free preparations $(P \geq 0.53$ for all comparisons, unpaired $t$-test), suggesting that sample physiology was well preserved.

Imaging activity in the entire volume of the third instar larval CNS generated large and information-rich data sets. We sampled the volume of the larval CNS using 25-50 million voxels and typically acquired 10 volumes per second ( 5 volumes per second per camera, 370 images per second), which produced sustained data streams of 500-1,000 MB per second for typically $1 \mathrm{~h}$. For each CNS with an estimated 12,000-15,000 neurons ${ }^{28}$, we thus recorded and analysed several terabytes of multi-view functional image data (see Methods section and Supplementary Methods).

Computational methods for whole-CNS image analysis. To address the wide spectrum of data processing and data analysis needs arising from our multi-view, whole-CNS functional imaging experiments, we developed a computational framework for hs-SiMView image data (see Fig. 1d, Methods section and Supplementary Data 2). This framework covers all essential image processing and data analysis tasks, including high-throughput lossless compression of the automatically detected image foreground, multi-view registration and fusion of the image foreground, local and global compensation of specimen drift across the time-lapse experiment, computation of a $\Delta F / F$ representation of the functional image data, automated detection and classification of fictive behaviours, and high-resolution mapping of activity timing relationships across the CNS during fictive behaviours. The latter two data analysis methods are explained in more detail in the context of the respective biological analyses presented in the following sections. Further details are provided in the Methods section.

We furthermore implemented a strategy for quantitative comparison of whole-CNS activity timing maps across multiple nervous systems. This method takes advantage of existing nonlinear image registration techniques for neural tissues 29,30 to create a reference template of the Drosophila larval nervous system. Using this template, we spatially registered and compared whole-CNS activity maps obtained from different specimens.

For efficient large-scale hs-SiMView data management, our framework automatically determines the geometry of the nervous system from multi-view image data and stores only voxels located in the image foreground containing the specimen, using our custom block-based KLB file format for lossless data compression. These algorithms reduce data size $>10$-fold.

All of our software modules are highly memory efficient and were designed for processing multi-terabyte data sets in a high-throughput setting. As a result of these design goals, all computations can be rapidly performed on a single computer workstation. The open source code and documentation of all software modules are provided in Supplementary Data 2.

Mapping whole-CNS activity patterns during fictive behaviours. On first inspection, large numbers of neurons and neuropil regions throughout the CNS showed complex activity patterns during fictive forward and backward crawling. As a first step towards understanding how higher-order control centres (brain lobes and SOG) coordinate the activity of primary larval motor centres (thorax and abdomen), we examined activity timing relationships between presumed higher-order regions and motor centres in 

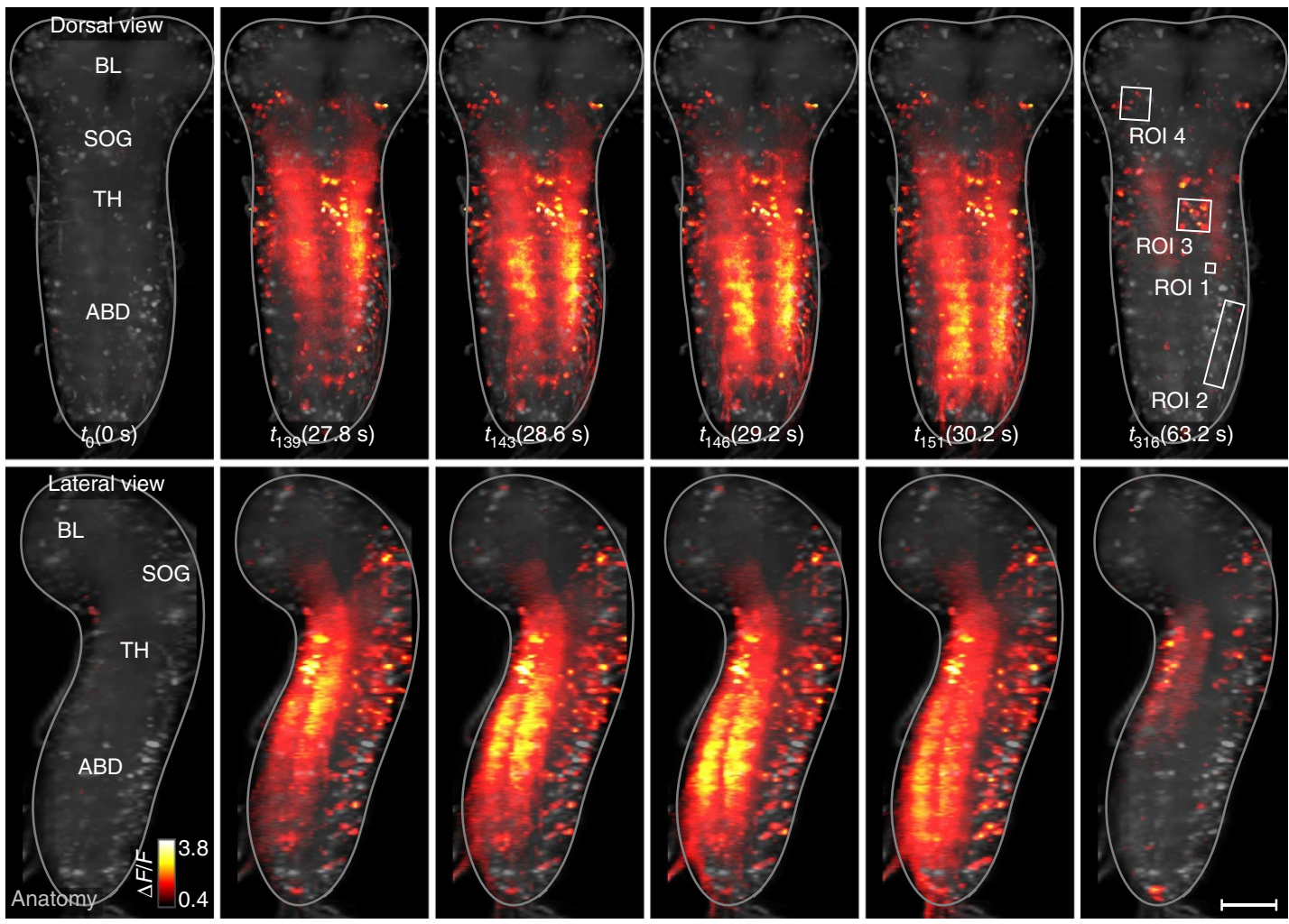

b
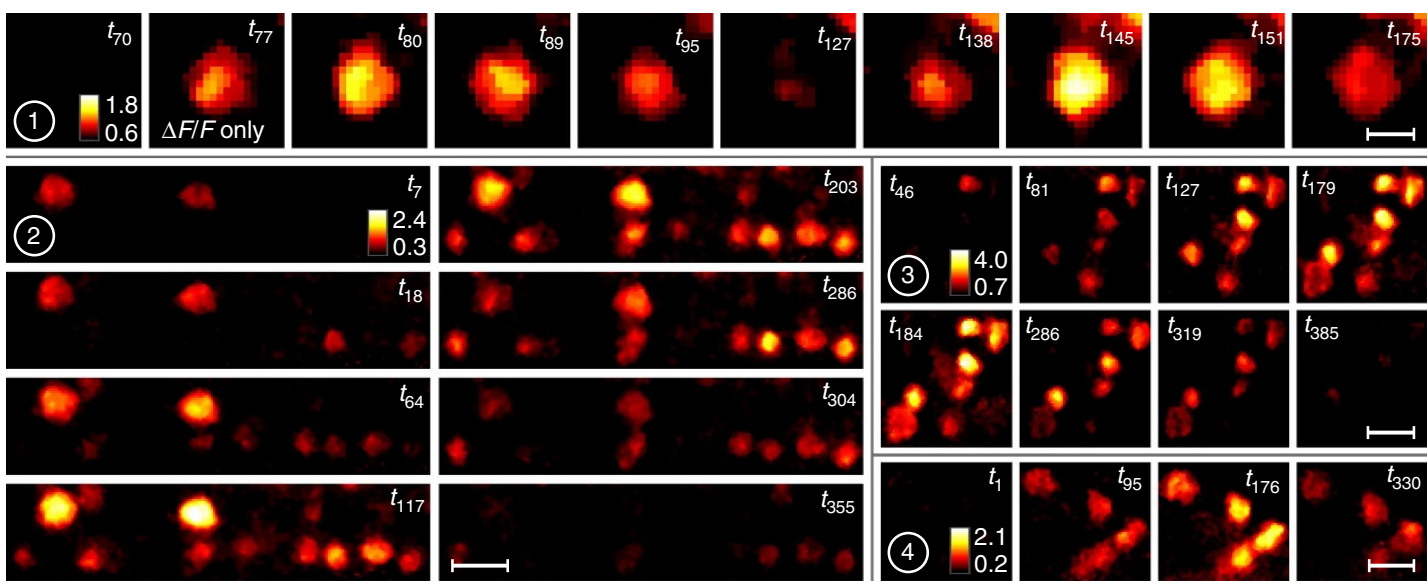

Figure 2 | Whole-CNS functional imaging of the isolated Drosophila larval nervous system. (a) Whole-CNS functional imaging at $5 \mathrm{~Hz}$ of a Drosophila third instar larval CNS expressing 57C10-GAL4,UAS-GCaMP6s, using hs-SiMView light-sheet microscopy. Imaging was performed with one-photon excitation at $488 \mathrm{~nm}$, maintaining a constant imaging speed of 370 frames per second (491 MB per second) for a period of $1 \mathrm{~h}$. Image panels show maximum-intensity projections of $\Delta F / F$ (colour look-up-table) and CNS anatomy (grey, gamma-corrected GCaMP6s baseline fluorescence) from dorsal (top) and lateral (bottom) views, for six time points during a backward locomotor sequence. Outline indicates CNS boundary. Longer image

sequences from this data set are shown in Supplementary Movies 1 and 2. Complementary data sets recorded with two-photon excitation are shown in Supplementary Movies 3-5. (b) Image sequences showing changes in $\Delta F / F$ for cell bodies in optical sections taken from ROIs in abdomen (ROIs 1 and 2), thorax ( $R O I$ ) and brain ( $R O I 4)$ indicated by white rectangles in panel a. ROI 1 shows an example of rapid changes in $\triangle F / F$ across two locomotor waves. ROIs 2-4 show examples of slow changes in $\triangle F / F$ across a bout of locomotor waves. Images are median filtered. ABD, abdomen; BL, brain lobes; SOG, suboesophageal ganglion; TH, thorax. Scale bars, $5 \mu \mathrm{m}$ (b, ROI 1), $10 \mu \mathrm{m}$ (b, ROls 2-4) and $50 \mu \mathrm{m}$ (a).

Drosophila larvae. Specifically, we wanted to determine whether we could identify regions or individual cells in higher-order centres that exhibit distinct and robust activity signatures for specific types of locomotor behaviours or during specific phases of a motor programme.

The larval CNS produced multiple types of segmentally coordinated motor activity. We decided to focus on forward and backward fictive crawling as they have the clearest relation to larval behaviour. To automatically detect and distinguish forward and backward waves, we used principal component analysis (PCA) for dimensionality reduction of $\Delta F / F$ signals extracted from abdominal neuropil regions (Fig. 3i). Based on features of these trajectories through PCA space, we systematically extracted event times for forward and backward waves in each experiment (see Fig. 3j, Methods section and Supplementary Data 2). By generating manual ground truth annotations for two complete data sets, we determined that our computational module for detecting and classifying motor activity produced, on average, 
a

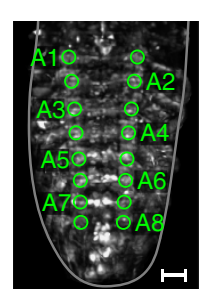

b
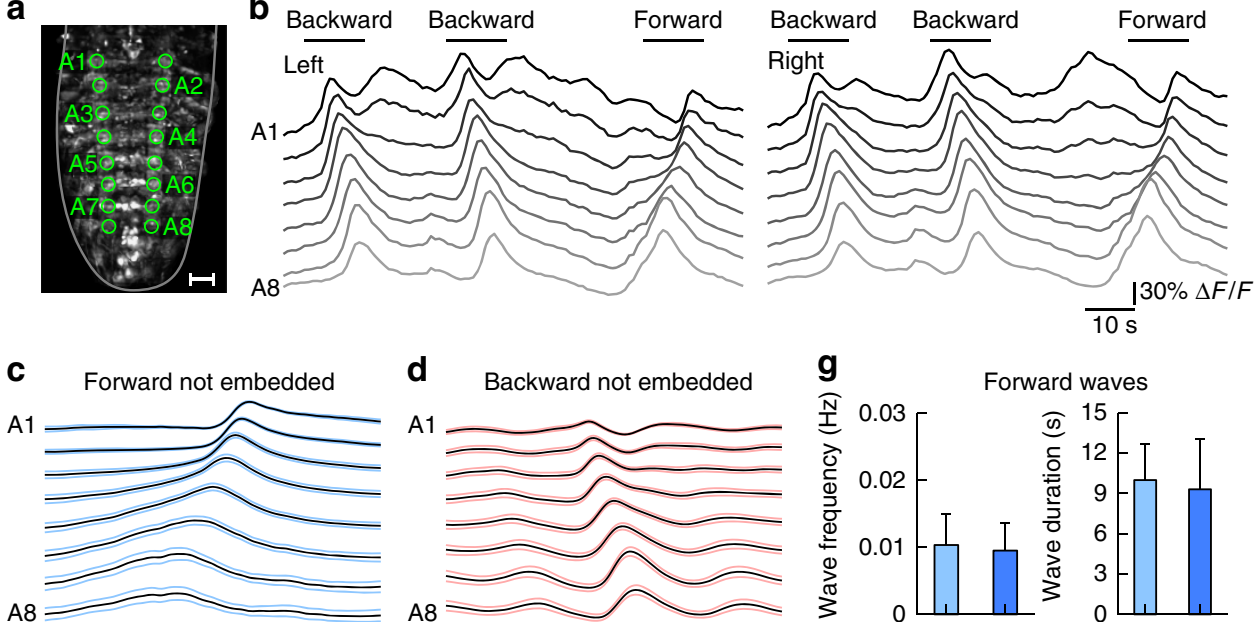

d
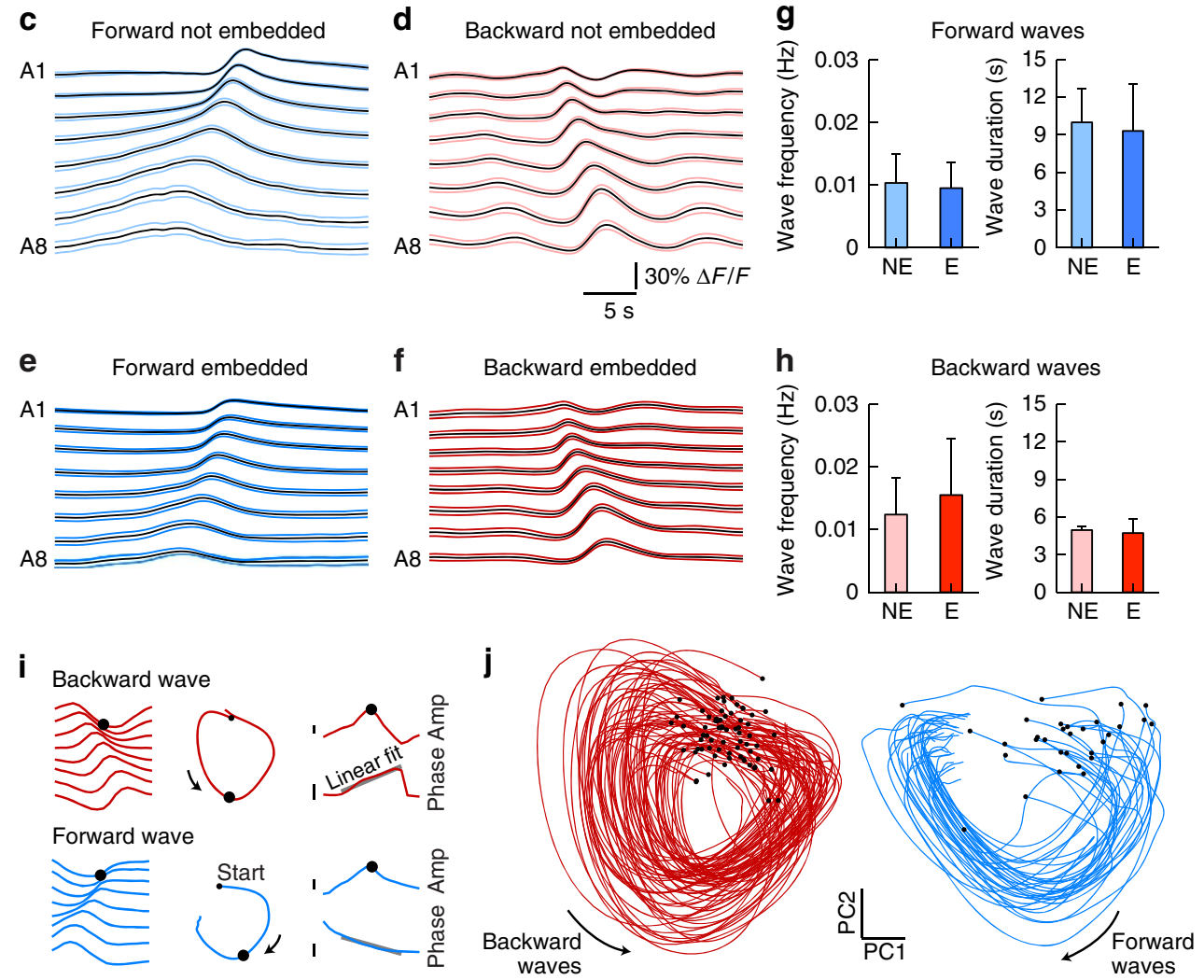

Figure 3 | Fictive locomotion in the Drosophila larval CNS. (a) Neural activity in all VNC hemisegments was captured using 16 3D ROls. Segments are labelled as A1-A8 from anterior to posterior. (b) Activity in hemisegments during example locomotor waves (two backward and one forward), shown separately for the left and right sides of the VNC. Traces show $\triangle F / F$. Shading indicates segments A1-A8. (c,d) Average forward (c) and backward (d) waves in a CNS in physiological saline (black: average, light blue: s.e.m.; $n=20$ waves). The plots show $\Delta F / F$ averaged over left and right sides in a representative preparation. (e,f) Same as in $\mathbf{c}, \mathbf{d}$, but for an isolated CNS embedded in agarose ( $n=20$ waves), which represents the sample preparation used for imaging with hs-SiMView microscopy. ( $\mathbf{g}, \mathbf{h})$ Average frequency and duration of forward waves ( $\mathbf{g}$ ) and backward waves (h) in unembedded (NE; $n=5$ preparations) and embedded preparations ( $E ; n=6$ preparations). Error bars show s.d. No significant differences in wave frequency or duration were observed between embedded and unembedded preparations ( $P \geq 0.53$ for all comparisons, unpaired $t$-test). (i) Illustration of principles underlying our computational module for automated detection and classification of locomotor waves. Left panels: example backward and forward waves; traces show $\Delta F / F$, with the average response across hemisegments subtracted, to emphasize relative differences. Middle panels: in a 2D space recovered using PCA, locomotor waves are rotations in different directions. Right panels: amplitude (Amp) and phase are computed in the same 2D space; waves are detected as peaks in amplitude and linear ramps in phase. (j) Embedding of all detected waves from one specimen in the 2D space recovered by PCA. Each trace is a wave; left, backward; right, forward. Small black dots indicate wave start times. Scale bar, $25 \mu \mathrm{m}$ (a).

94.5\% correctly classified hits and $5.5 \%$ false alarms $(n=152$; see Methods section). We then used these event times as triggers and computed a mapping of activity timing to locations in the entire CNS for all data sets (see Methods section). This analysis uses machine learning to compute, on a voxel-by-voxel basis, the improvement in fit of a time-locked activity model over a flat response model during network events (Fig. 4a and Supplementary Data 2). On an intuitive level, this method provides a measure, per voxel, of when and to what extent activity increases in the locomotor event window. Such voxel-level information represents a useful complement to analyses based on annotated and segmented structures (presented in the next section), especially when manually vetted annotation is difficult owing to the scale or complexity of the data, and when neuronal response properties need to be mapped across diverse neuronal morphologies.

We used this activity timing analysis to first examine how activity propagates through the entire volume of the larval CNS during forward and backward fictive crawling. By plotting only the timing information provided by this analysis, activity progression can be charted and easily followed through the larval CNS through a large dynamic range of local changes in activity (Fig. 4b,c). Previous anatomical work has determined that dorsal regions of the larval VNC contain primarily motor neurons and 
premotor circuitry, whereas ventral regions of the VNC contain primarily sensory terminals and interneurons involved in sensory processing ${ }^{31,32}$. Given this anatomical organization, we predicted that waves of activity would be most prominent and wave propagation would be fastest in the dorsal regions of the larval VNC. Indeed, wave propagation during both forward and backward waves was faster in dorsal regions versus ventral regions (Fig. 4b and Supplementary Movies 9 and 10): during forward waves, propagation of peak activity from segments 8 to 3 was $29 \%$ faster dorsally versus ventrally (speed measured from median activity timing of $n=30$ waves); during backward waves, propagation of peak activity from abdominal segments 1 to 6 was $60 \%$ faster dorsally versus ventrally ( $n=70$ waves). Moreover, during forward waves, peaks in dorsal regions significantly preceded those in ventral regions in all abdominal segments $\left(P<10^{-5}\right.$, sign test, $n=30$ per segment); during backward waves, peaks in dorsal regions significantly preceded those in ventral regions in abdominal segments 8 through $3\left(P<10^{-13}\right.$ for all segments, sign test, $n=70$ per segment), whereas activity timing for the first two segments was indistinguishable in dorsal and ventral regions. Increase in activity in dorsal regions was higher than in VNC regions by a factor of $3.9 \pm 1.1$ during forward waves (mean \pm s.d., $n=227$ ) and by a factor of $3.1 \pm 1.2$ during backward waves (mean \pm s.d., $n=495$ ).

Interestingly, as activity propagated forward through the $\mathrm{VNC}$, activity also propagated from the SOG to the dorsal-most regions of the brain (Fig. 4b,c, left panels; Supplementary Movie 9). Statistical comparison of activity peak times confirmed that peaks in SOG regions significantly preceded those in ventro-caudal brain lobe regions $(P<0.001$, sign test, $n=30)$, which in turn significantly preceded those in dorsal brain lobe regions $(P<0.001$, sign test, $n=30)$.

Our timing analysis also revealed local hot spots with distinct activity patterns, in particular, in the thorax and SOG (Fig. 4c and Supplementary Movies 9 and 10). In order to resolve the fine temporal structure of activity in these regions of interest (ROIs), we extracted fluorescence traces from 24 ROIs within the thorax, SOG and brain lobes that corresponded to boundaries and distinct regions in the activity timing maps (Fig. 4d,e). Peak activity in ROIs corresponded to average timing assignments in the voxel map with a precision exceeding the temporal sampling of the image data $(0.15 \pm 0.14$ s, mean \pm s.d., $n=47)$, confirming that our activity timing maps provided accurate information on relative timing of events in different parts of the larval CNS not only at the voxel level but also at a regional level. Across our set of ROI annotations, we found bilaterally symmetric sites with activity peaks occurring during initiation (before peak in leading abdominal segment), execution (between A1 and A8 peaks) and/ or termination (after peak in final abdominal segment) phases of locomotor waves. Activity in SOG ROIs 1-5 (L/R) peaked during the execution phase of forward waves and was maximally suppressed during the execution phase of backward waves. Activity in thoracic ROIs 6-7 (L/R) was bimodal during backward waves, with peaks occurring during initiation and termination phases. Activity in thoracic ROIs $8-10$ (L/R) peaked during execution phases of both forward and backward waves. Activity in brain ROIs 11-12 (L/R) was strikingly different during forward and backward waves: activity peaked during the termination phase of forward waves, whereas bimodal activity with peaks during initiation and termination phases was observed for backward waves (Fig. 4e).

Large-scale profiling of single-neuron activity during behaviours. After mapping information flow through the CNS during forward and backward waves at a coarse level, we examined activity patterns at the level of individual neurons during these two fictive behaviours. We identified and annotated cell body locations in two preparations imaged with one-photon hs-SiMView (Fig. 5) and two-photon hs-SiMView (Supplementary Fig. 7), respectively.

During forward waves (Fig. 5b,c), most annotated cells in the VNC reached peak activity during wave propagation (defined as the time interval marked by peak activity in abdominal segments 1 and 8): 73\% of neurons reached peak activity during wave propagation, whereas $22 \%$ reached peak activity after the end of wave propagation $(n=100)$. A small fraction $(5 \%)$ of neurons showed two activity peaks separated by a period of quiescence as forward waves propagated through abdominal segments. In the SOG and brain lobes, activity patterns were more diverse, with a large number of cells becoming active at various phases of the motor programme: $10 \%$ of neurons reached peak activity before the start of wave propagation, $27 \%$ during wave propagation and $40 \%$ after the end of wave propagation $(n=60)$. Interestingly, a relatively large fraction of annotated neurons in SOG and brain lobes (23\%) showed two activity peaks separated by a period of quiescence as forward waves propagated through abdominal segments.

During backward waves (Fig. 5b,c), neurons in the VNC were largely active with locomotor waves in phase relationships similar to those observed for forward waves: $68 \%$ of neurons reached peak activity during wave propagation, $24 \%$ after the end of wave propagation and $7 \%$ showed two activity peaks separated by a period of quiescence during wave propagation $(n=100)$. In contrast, in the SOG and brain lobes, activity patterns were quite distinct from those observed during forward waves: the vast majority of cells (72\%) were active after the end of the backward wave propagation, and $8 \%$ of cells were active before the initiation of the backward wave $(n=60)$. Thirteen per cent of neurons showed two activity peaks separated by a period of quiescence as the backward wave propagated through abdominal segments.

A comparison of the classification of peak activity in each neuron during forward and backward waves (Fig. 5c) revealed conserved activity signatures for in total $74 \%$ of annotated neurons in abdominal segments $(n=100)$ and $42 \%$ of annotated neurons in the brain lobes and SOG $(n=60)$.

Neurons in the brain identify type and state of motor programs. Our initial mapping of activity timing throughout the CNS identified small regions within the SOG that showed activity that was strongly synchronized with activity in distant abdominal regions. Closer examination revealed that these regions were often identifiable as cell bodies. However, variations in CNS orientation and shape across preparations made it difficult to assay whether the locations of cell bodies were conserved from animal to animal. To address this problem, we constructed a global CNS anatomy template using GCaMP image data obtained from six nervous systems, following the symmetric group-wise normalization approach $(\mathrm{SyGN})^{30}$, and registered all image data and activity timing maps to this template using B-spline diffeomorphic registration $^{32}$ (see Fig. 6a and Methods section). We used several orthogonal approaches to determine registration accuracy (see Methods section). The average volume overlap between registered, individual nervous systems and global template was $95.2 \pm 1.1 \%$ (mean \pm s.d., $n=6$ ). The average distance between corresponding, manually annotated landmarks in the registered nervous systems was $4.5 \pm 5.9 \mu \mathrm{m}$ (mean \pm s.d., $n=150$ ) for landmarks in the VNC and $16.3 \pm 8.6 \mu \mathrm{m}$ (mean \pm s.d., $n=60$ ) for landmarks in the brain (Fig. 6b,c).

In three spatially registered nervous systems that each yielded good statistics for locomotor waves ( $n \geq 30$ per specimen), we 
identified four neurons located ventro-laterally on either side of the SOG with interesting features; across the three preparations, these neurons had highly conserved cell body positions (with an average pairwise distance of $13.8 \pm 5.8 \mu \mathrm{m}$ across preparations, mean \pm s.d., $n=24$ cell pairs; Fig. $7 \mathrm{a}-\mathrm{c})$, strong bilateral symmetry and distinct patterns of activity timing (Fig. $7 \mathrm{~d}-\mathrm{f}$ ).

Type 1 neurons were located in the posterior SOG (Fig. 7d). During forward waves, their activity slowly ramped up over a period of several seconds then peaked shortly after the wave had passed the first abdominal segment, marking the end of forward wave propagation $\left(P<10^{-7}\right.$, sign test, $\left.n=60\right)$. During backward waves, activity in these cells slowly ramped up, at a rate similar to their initial behaviour during forward waves, but activity then remained elevated for $\sim 5 \mathrm{~s}$ following the termination of the wave (Fig. 7e).

Activity in type 2 and 3 neurons during forward waves was similar to that observed in type 1 neurons; however, during backward waves, activity slowly decreased over a period of several seconds, then rebounded after the locomotor wave had passed the first three abdominal segments $\left(P<10^{-11}\right.$, sign test, $n=264$; Fig. 7e).

Activity in type 4 neurons during forward waves ramped up and peaked when the wave reached abdominal segment A6 $\left(P<10^{-5}\right.$, sign test, $\left.n=60\right)$, then quickly decreased and rebounded when the wave had passed the first abdominal segment $\left(P<10^{-12}\right.$, sign test, $\left.n=60\right)$. During backward waves, activity in type 4 neurons largely tracked activity observed in abdominal segment A8 (Fig. 7e).

Similar results were obtained in corresponding cell body pairs during forward waves in two other preparations (Fig. 7f). Each cell showed robust, reproducible responses over multiple trials and was information-rich in that the dynamics of activity within each cell reflected both the direction and progression of locomotor waves through the VNC (Fig. 7e,f).

\section{Discussion}

The imaging framework described here represents the first method capable of recording neural activity at near cellular resolution throughout the CNS of a higher invertebrate. By applying this framework to the Drosophila larval CNS, we found that representations of fictive locomotor activity are present in presumed higher-order centres at both a regional level and at the level of individual neurons, and that these representations change depending on the mode of fictive locomotion.

At the foundation of our approach is our new hs-SiMView multi-view light-sheet microscope capable of high-speed functional imaging with one- or two-photon excitation. Whole-CNS calcium imaging in a nervous system as large as that of Drosophila critically required the design of a microscope with substantially improved temporal resolution, from tens of seconds offered by state-of-the-art multi-view light-sheet microscopy to a few tenths of seconds achieved in hs-SiMView. To enable the reproduction of the imaging technology presented here, we provide detailed technical drawings for all critical custom parts of the hs-SiMView microscope (Supplementary Data 1). From a technical perspective, we found that multi-view imaging at high spatiotemporal resolution is crucial to capture the propagation of activity through the entire Drosophila CNS and to systematically identify individual neurons with distinct activity patterns during fictive behaviours. At the same time, the low light exposure and long-term imaging capability provided by our method are essential to collect enough data from a single specimen to build statistically meaningful whole-CNS activity maps. When comparing whole-CNS functional imaging with one-photon and two-photon excitation, we found that the former approach offers superior imaging speed and experiment length (Supplementary Fig. 8), whereas the latter offers superior signal-to-background levels and thereby simplifies large-scale identification of cell bodies (Supplementary Fig. 7).

In order to use this imaging technique to study neural network dynamics at the scale of the entire CNS, we developed a suite of computational tools. In previous work, we and others devised tools for the analysis of large-scale functional imaging data $^{7,8,16,33-35}$. Here, we explored several new directions essential to tackle the challenges encountered when analysing multi-view calcium imaging data in general and motor activity at the scale of an entire CNS in particular. We present approaches for processing and management of multi-view calcium imaging data recorded with hs-SiMView microscopy, automated detection of coordinated motor activity and high-resolution mapping of functional relationships across the CNS during fictive motor behaviours. The methods facilitate efficient processing and analysis of multi-terabyte hs-SiMView image data sets on a single computer workstation. At the same time, many of our tools are also capable of leveraging the strengths of computer clusters, for example, for rapid exploration, screening and comparison of different data processing strategies. Our software modules, including open source code and a software user guide, are provided as Supplementary Data 2.

The overall strength of our combined experimental/ computational approach is that it enables the simultaneous, unbiased examination of activity relationships among disparate but functionally connected CNS regions. Thereby, whole-CNS activity patterns are mapped in a single preparation, providing information on multiple fictive behaviours and with cellular

Figure 4 | Mapping whole-CNS activity during locomotor behaviours. (a) Illustration, for data from one example voxel, of $\Delta F / F$ fits performed for purpose of mapping activity timing during locomotor wave windows. Plots show activity traces in all waves sampled (thin, grey), the mean of these traces (black), the rectangular time-based function fit (red) and the flat, time-independent fit (blue). In this example, the time-based fit is much better than the flat fit. This analysis is performed independently for all voxel locations in the image volume. (b,c) Whole-CNS activity timing maps for forward and backward waves (b, dorsoventral slices; $\mathbf{c}$, lateral slices). The statistic shown in a was used to capture relative timing of activity across all detected wave events in one specimen (forward: $n=30$, backward: $n=70$ ). Intuitively, this map shows, for each part for the $C N S$, the time during locomotor wave windows when activity increases. Arrows in $\mathbf{b}$ mark relative progression of locomotor waves on dorsal/ventral sides of the VNC (ascending numbers). Forward and backward wave window sizes were defined as $[-10 \mathrm{~s}, 10 \mathrm{~s}]$ and $[-6 \mathrm{~s}, 2 \mathrm{~s}]$ (centred on waves in VNC) to ensure wave propagation was captured throughout VNC and overlap of events was avoided. (d) Lateral slices from 3D whole-CNS activity timing map of fictive forward locomotor waves ( $n=30$ ), with ROIs outlined in white and black (bottom right panel). ROIs include bilaterally symmetrical regions in SOG with increased activity occurring at beginning of forward locomotor waves (1L/R-5L/R), regions in the thorax and at boundary between thorax and abdomen with increased activity occurring during $(8 \mathrm{~L} / \mathrm{R}-10 \mathrm{~L} / \mathrm{R})$ or towards the end $(6 \mathrm{~L} / \mathrm{R}$ and $7 \mathrm{~L} / \mathrm{R})$ of forward waves, and regions at dorsal end of brain lobes with increased activity occurring after execution of forward waves (11L/R and 12L/R). (e) $\Delta F / F$ traces of ROIs $1 \mathrm{~L}-12 \mathrm{~L}$ (left two columns) and 1R-12R (right two columns) indicated in panel $\mathbf{a}$, during forward $(n=30)$ and backward $(n=66)$ locomotor waves. Four backward waves were excluded to avoid temporal overlap. For reference, $\Delta F / F$ traces of segments $A 1-A 8$ in the VNC are shown below data for ROls 1L/R-12L/R. Dark lines indicate average activity, light areas indicate s.e.m. $L$, left; $R$, right; $A B D$, abdomen; $B L$, brain lobes; SOG, suboesophageal ganglion; $T H$, thorax. Scale bars, $50 \mu \mathrm{m}$ (b-d). 
resolution for a large fraction of the CNS. In order to identify anatomical regions and individual neurons with conserved activity patterns from these whole-CNS activity maps, we furthermore took advantage of methods for spatial registration of neural tissues ${ }^{30,32}$. By combining nonlinear image registration techniques with independent genetic expression systems in
Drosophila, we implemented and validated a strategy for the quantitative comparison of whole-CNS activity maps across multiple nervous systems. This approach is robust even in the face of relatively large disparities in tissue size, orientation and topology, and may therefore present a useful strategy that could be applied to other animal models as well. a

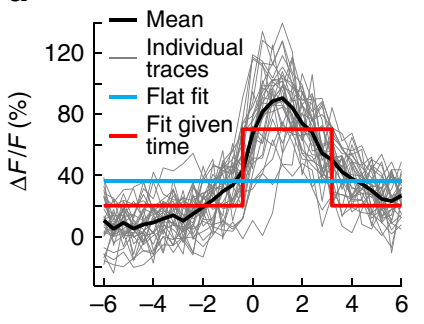

Time relative to wave detection (s) b Forward waves

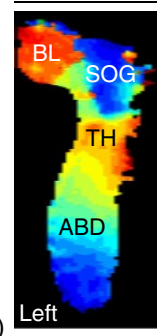

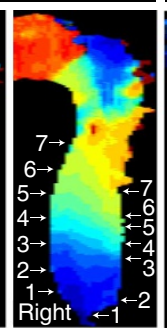

Backward waves

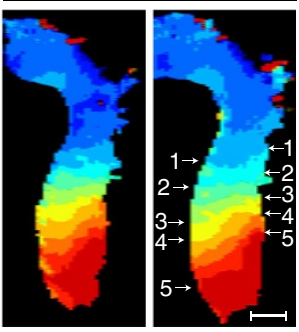

c

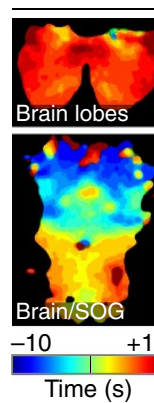

Forward waves
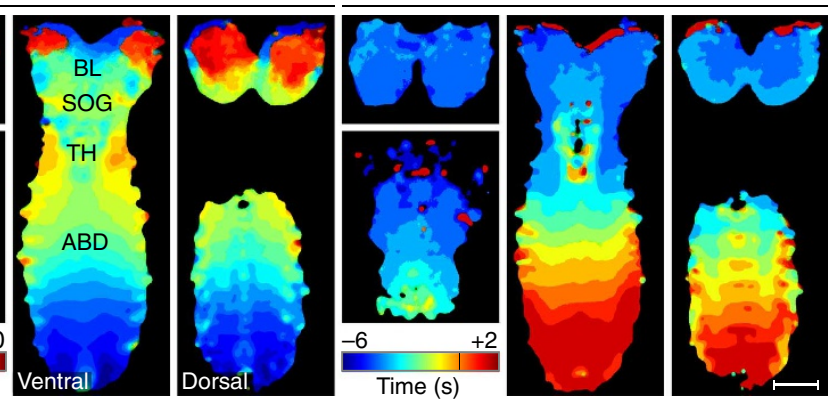

d
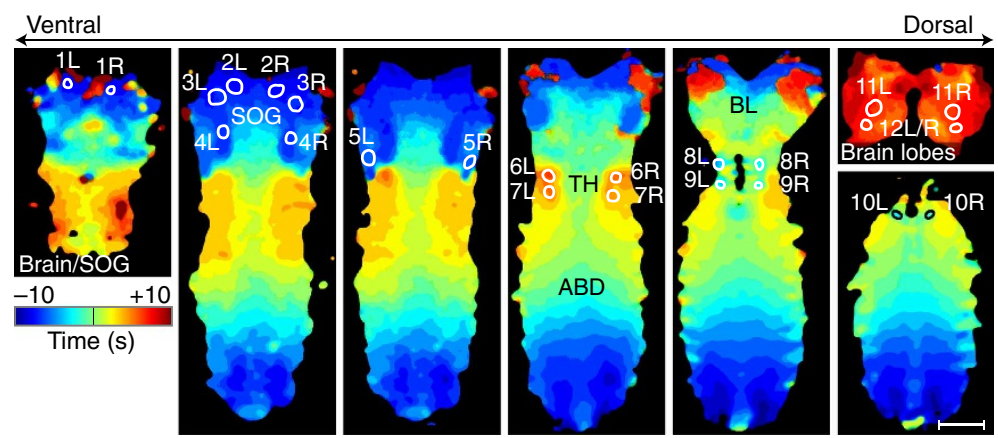

e
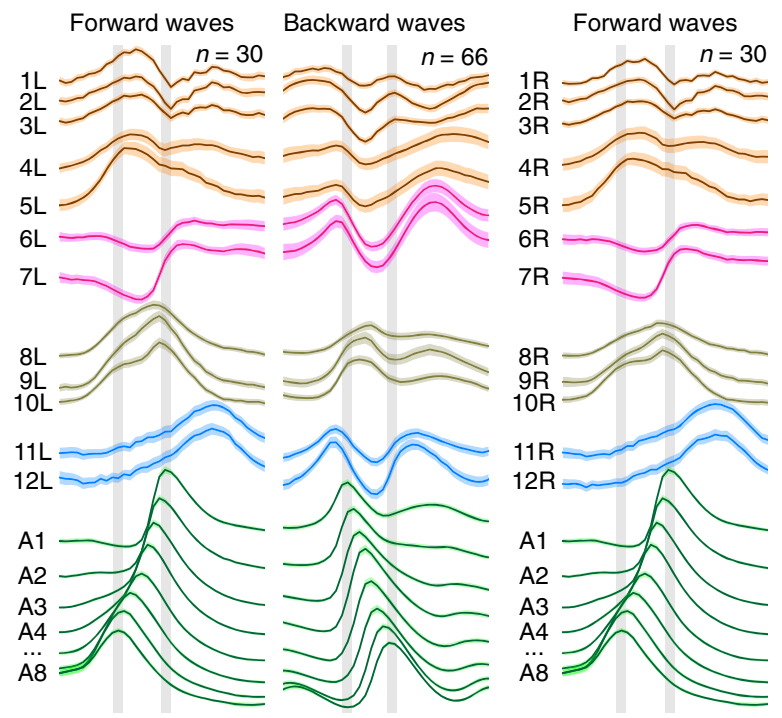

Backward waves
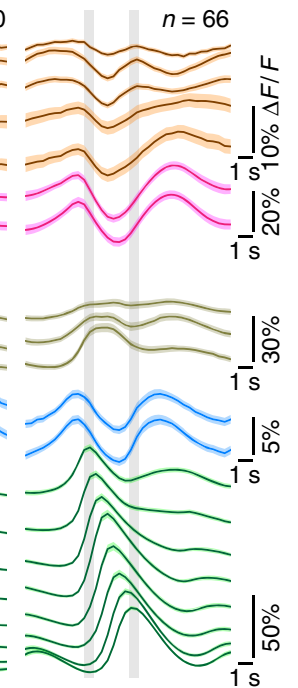
a

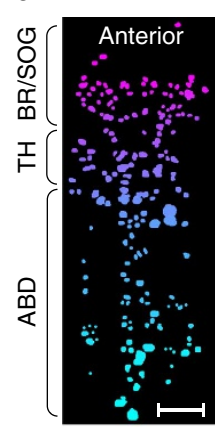

Soma index 200

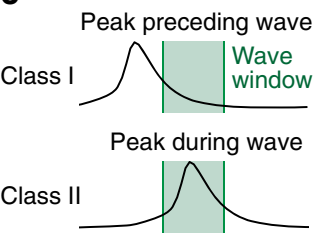

Peak succeeding wave

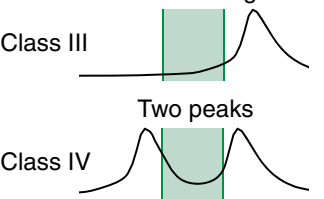

b Forward Backward Forward Backward

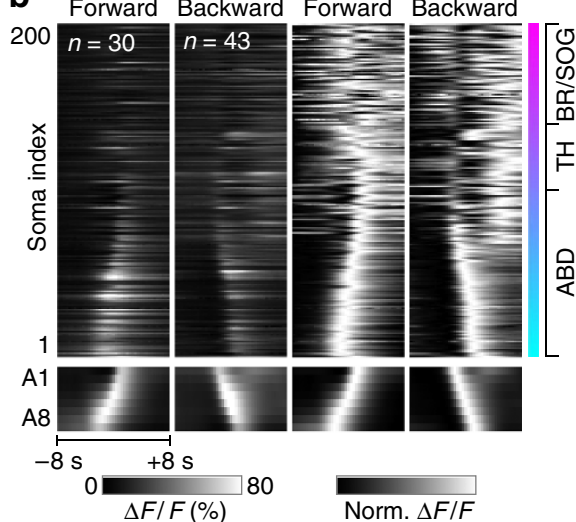

\section{8} 要 T waves. This raises the possibility that the SOG as a region may play a role in the initiation or monitoring of forward locomotion, whereas dorsal brain regions may play a role in its termination. At a coarse spatial level, activity in the SOG did appear to have distinct overall timing relationships relative to other CNS regions. It is important to note, however, that local regions within the SOG showed very strong but heterogeneous activity patterns during motor programs; likewise, thoracic regions also contained several such 'hot spots'. Based on their distinct timing relationship with the motor programs, some of these regions could be involved in the initiation, termination and/or monitoring of motor activity ${ }^{36,37}$. Interestingly, these results also make specific predictions about long-range coarse connectivity between CNS regions. Two testable hypotheses for future study are that strong functional connections exist between the larval SOG and posterior abdominal segments and between dorsal regions of the brain and lateral thoracic regions.

Our analyses were also able to identify single neurons within the SOG exhibiting patterns of activity that, to varying degrees, were representative of the type and temporal state of motor programs. Neurons that encode features of motor behaviours (that is, velocity and acceleration of limbs, as well as activation of muscles) have been identified in motor control centres of the vertebrate brain ${ }^{38}$. Descending neurons in the SOG of the leech, Hirudo medicinalis, also exhibit patterns of activity that reflect distinct behavioural states (for example, crawling). When stimulated, these neurons trigger changes in body posture and behavioural state ${ }^{39}$; however, behavioural choice in the leech appears to result from both dynamics of neuronal populations and the actions of a select few neurons that can strongly influence decision making ${ }^{40}$. The variety of candidate neurons, SOG regions and brain lobe areas with network state-related activity profiles discovered in our systematic search may thus provide a foundation for future functional studies aimed at understanding how the larval CNS generates and selects between distinct motor patterns.

In Drosophila larvae, a coordinated effort aimed at constructing a complete connectome is currently underway ${ }^{41}$, and the exciting prospect of coupling imaging-based identification of candidate neurons, and whole-CNS imaging in general, to connectomics data is thus on the immediate horizon. Integrating the circuit maps resulting from this work with the functional imaging assay presented here may allow for detailed examination of how behaviour is produced as a function of both neural network architecture and dynamics of activity within interconnected neurons.

Two complementary directions for further research are centred on technology development. On the one hand, further improvement of spatial resolution will generally be desirable, in particular, in applications that require comprehensive single-cell coverage throughout the third instar larval CNS. Possible strategies to this end include the integration of wide-field adaptive optics in the microscope's detection $\operatorname{arms}{ }^{42}$, and automated, adaptive rotation and translation of light sheets to compensate for local specimen-induced light refraction in the illumination process ${ }^{43}$. Moreover, dual use of the microscope's four optical arms for light-sheet illumination as well as fluorescence detection may present a very effective approach to improve axial resolution ${ }^{4}$, and could even enable the development of large-scale functional imaging assays with isotropic spatial resolution in the future. On the other hand, to 
a
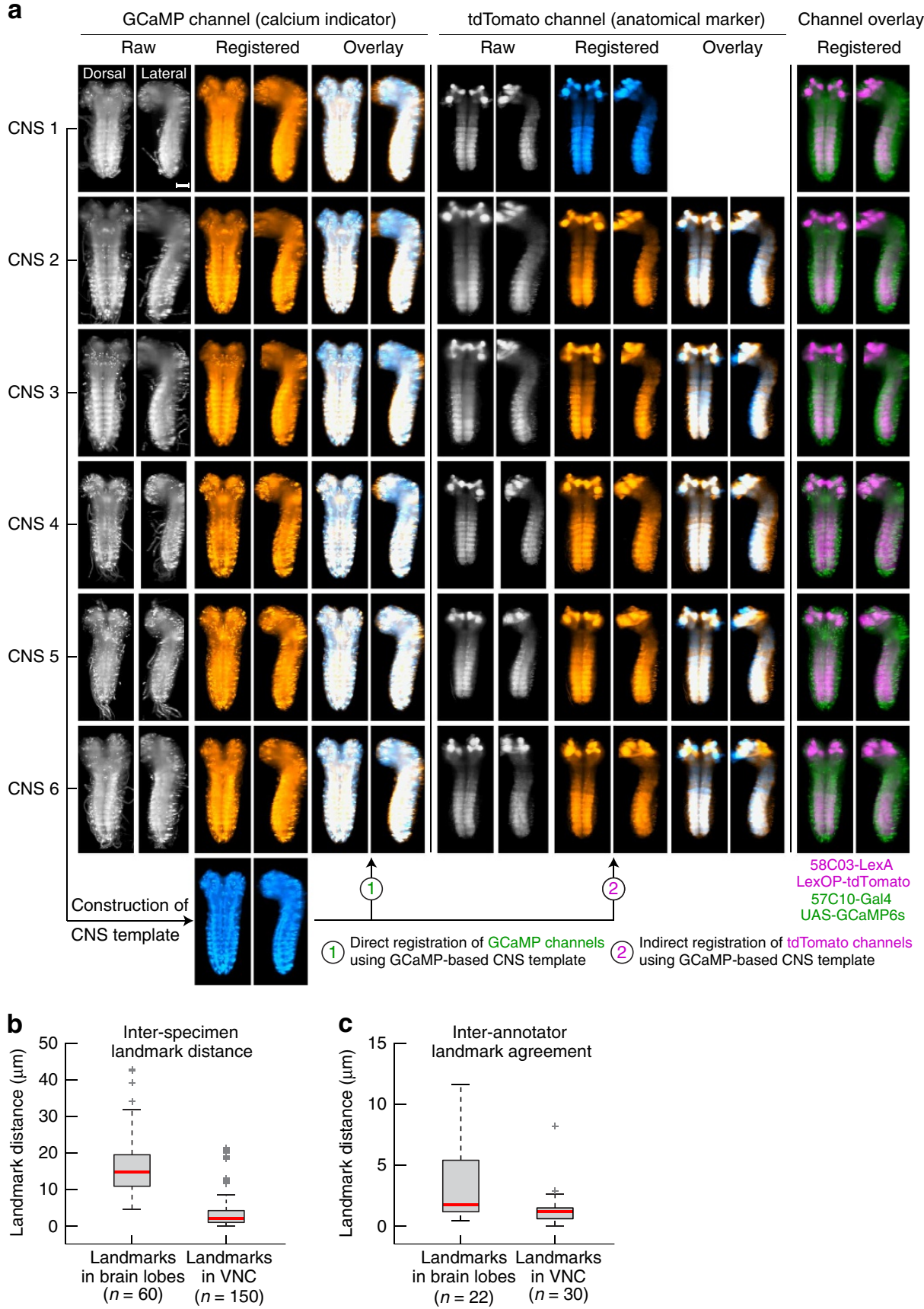

Figure 6 | Spatial registration of nervous systems. (a) All CNS explants used for functional imaging expressed GCaMP panneuronally (57C10-

GAL4,UAS-GCaMP6s) and tdTomato in anatomically defined regions within the larval CNS (58B03-LexA,LexOP-tdTomato; expression in mushroom bodies and neuropil regions). Columns 1-3 show maximum-intensity projections of unregistered GCaMP images (column 1), spatially registered GCaMP images (column 2) and respective overlays (column 3) for six different Drosophila third instar larval CNS explants. To register nervous systems, we first constructed a CNS template (shown at the bottom of column 2) from GCaMP reference image stacks representing each of the six independent time-lapse experiments (see Methods section). We then registered image data from each experiment to the CNS reference template using nonlinear spatial registration techniques (see Methods section). Overlays of registered image stacks (orange) and CNS reference template (blue) in column 3 show good anatomical correspondence. Since the CNS reference template used for spatial registration of all nervous systems was constructed exclusively from GCaMP images, we used the tdTomato channel (column 4) to evaluate how well an independent expression pattern can be overlaid across multiple nervous system using our registration workflow. In this control analysis, spatial correspondence depends on quality of the spatial registration and biological variability of the expression pattern. Registration of the tdTomato channels of the six explants to the CNS reference template (column 5) resulted in good overlap of expression patterns in CNS 1-5 (column 6), which appeared to highlight the same biological structures in the CNS. The expression pattern in CNS 6 looked qualitatively different from the others, possibly due to transvection of the LexAop-myr::tdTomato transgene ${ }^{51}$. (b) To quantify registration accuracy, two annotators independently determined the 3D positions and relative distances of several CNS landmarks (including landmark locations in abdominal segments and mushroom bodies) in the spatially registered specimens. VNC landmarks were annotated in GCaMP images (obtained by direct registration), whereas brain lobe landmarks were annotated in tdTomato images (indirect registration). The latter measurements thus reflect both accuracy of the registration procedure and biological variability. (c) Inter-annotator agreement of landmark positions independently annotated in the same nervous systems. Scale bar, $50 \mu \mathrm{m}$ (a). 


\section{a}

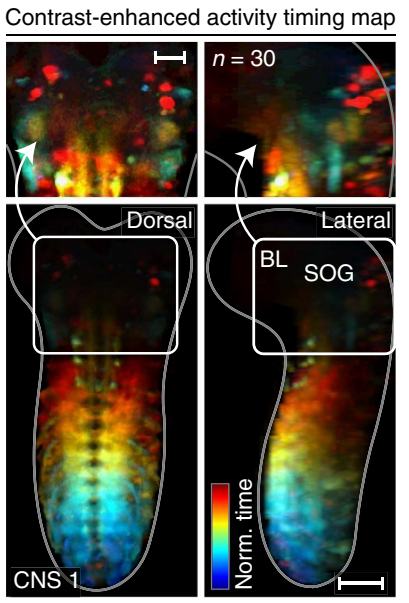

Whole-CNS activity timing map
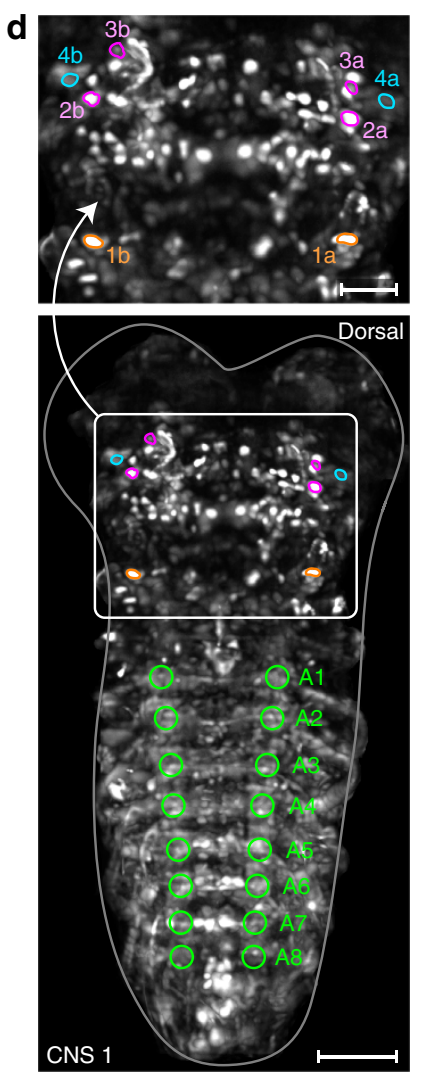

b

Registered brain segmentation maps
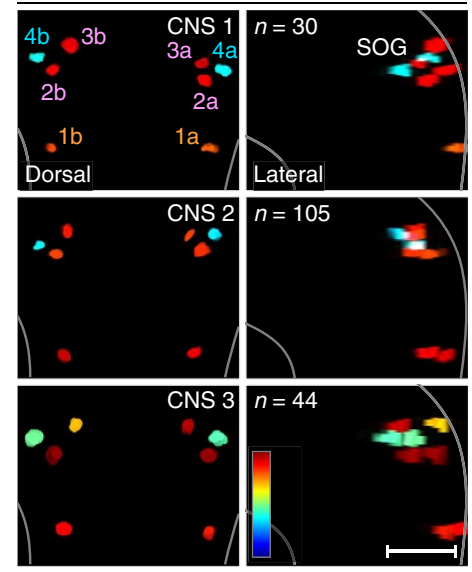

$\mathbf{c}_{\mathrm{N}, \mathrm{H}}$

Multi-CNS segmentation overlay

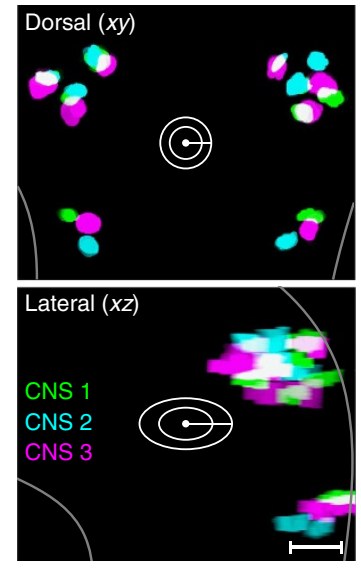

e Forward waves (CNS 1)

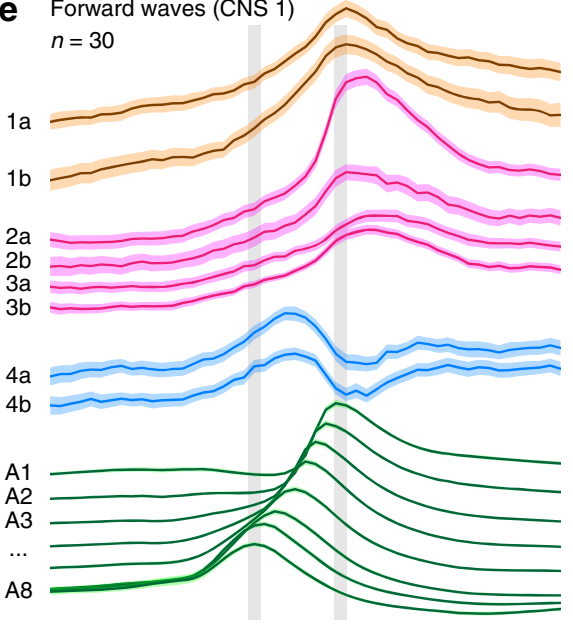

f Forward waves (CNS 2)

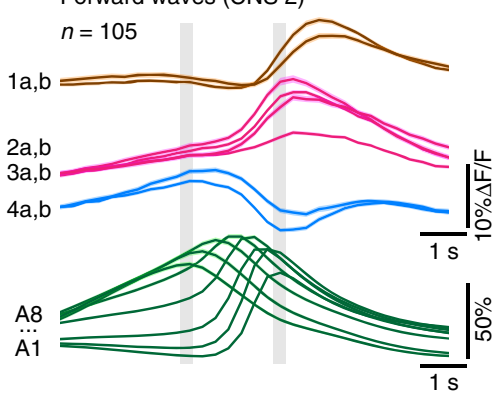

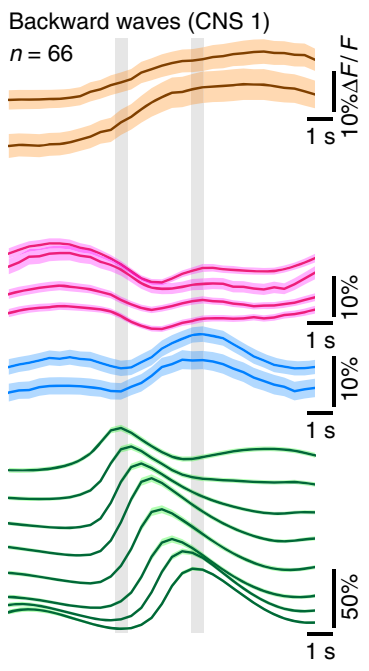

Forward waves (CNS 3)

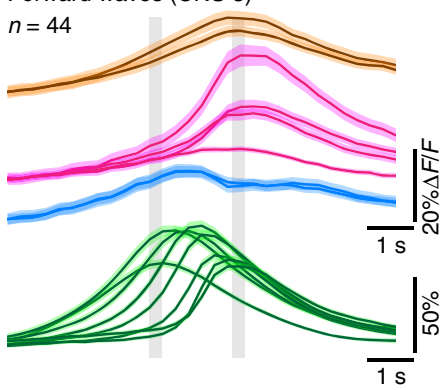

Figure 7 | Neurons in the brain identify type and state of motor programs. (a) Dorsal (left) and lateral (right) maximum-intensity projections of wholeCNS activity timing map for forward waves $(n=30)$. Colour encodes time point of maximum activity. Luminance encodes improvement in activity fit given time (see Methods section). Top panels show enlarged views of brain/SOG, using a luminance look-up-table restricted to dynamic range of data in this region for better visibility. (b) Top: maximum-intensity projections of eight manually segmented single-soma ROls from brain/SOG region highlighted in a. Middle and bottom: segmented brain/SOG maps from two additional specimens (CNS 2 and CNS 3), based on 105 and 44 forward waves, respectively. All three nervous systems were spatially co-registered as described in Methods section. Colour code as in $\mathbf{a}$. (c) Overlay of the three brain/SOG segmentation maps shown in $\mathbf{b}$, demonstrating close spatial correspondence of segmented soma locations. Colours indicate specimen identity (green/cyan/magenta: CNS 1/2/3). White circles ( $x y)$ and ellipses $(x z)$ in centre of images indicate average accuracy of the spatial registration procedure (inner circle/ellipse: mean, outer circle/ellipse: mean +1 s.d.). Accuracy in brain is $7.8 \pm 4.6 \mu \mathrm{m}$ (mean \pm s.d., $n=60$ ) along $x$ - and $y$-directions and $13.0 \pm 9.5 \mu \mathrm{m}$ (mean \pm s.d., $n=60$ ) along $z$-direction. (d) Dorsal maximum projection of deconvolved image data of CNS 1 (serving as anatomical reference), superimposed with manually segmented single-soma ROls shown in $\mathbf{b}$ (top) and hemisegment ROIs for tracking locomotor wave propagation in the VNC (green, segments are labelled as A1-A8). (e) Activity traces for eight manually annotated single-soma regions in CNS 1 shown in $\mathbf{b}$ (top) and $\mathbf{d}$, averaged over all forward (left) and backward (right) time windows. Dark lines indicate average activity, light areas indicate s.e.m. (forward: $n=30$, backward: $n=66$ ). Vertical grey bars indicate time of peak activity in A1 and A8. (f) As in e, but for forward waves in CNS $2(n=105)$ and CNS $3(n=44)$. Data for backward waves are not shown due to low statistics for this fictive behaviour in these two specimens ( $n=6$ and 5 , respectively). BL, brain lobes; SOG, suboesophageal ganglion. Scale bars, $25 \mu \mathrm{m}$ (a, top; $\mathbf{c}$ and $\mathbf{d}$, top), $50 \mu \mathrm{m}$ (a, bottom; b and $\mathbf{d}$, bottom). 
make the most of this new technology, it will be important to also develop strategies for imaging activity in large neural networks simultaneously with optogenetic manipulation. Thereby, the functional role of individual neurons could be investigated in the network context. Moreover, functional imaging assays could be further enriched by taking advantage of advances in voltage indicators ${ }^{45}$, availability of multiple independent genetic expression systems $s^{46-49}$ and the wealth of cell-type specific driver lines that are becoming available for Drosophila ${ }^{18,19}$.

The method presented here for the isolated larval CNS can be readily adapted to other developmental stages, including Drosophila pupae and adults, and may also be suitable for in vivo whole-animal functional imaging of Drosophila embryos and first instar larvae. We note that this latter approach introduces new challenges in the domain of image processing and data analysis, and will greatly benefit from the development of spatial registration techniques for moving specimens and specimens with dynamically changing topologies. Our hs-SiMView microscope and computational image analysis framework should also be suitable for the study of other biological model organisms. Possible models include not only other invertebrates with a compact CNS but also vertebrates, such as zebrafish embryos and larvae, that benefit greatly from multiview imaging with light-sheet microscopy ${ }^{25}$, as well as partial CNS explants of animal models with larger nervous systems. The main limitation of our imaging technique concerns sample size, which is constrained by the travel range of the piezo stages and the need for optical access to the sample from multiple directions. The maximum volume accessible with the microscope configuration presented here is $800 \times 800 \times 250 \mu^{3}$. Larger volumes can be covered by simply switching to different detection objectives and piezo stages with longer travel range; for example, using C-Apochromat $\times 10 / 0.45$ objectives (Carl Zeiss) in combination with P-629.1CD Hera piezo stages (Physik Instrumente, Karlsruhe) the multi-view imaging volume can be increased to $1,300 \times 1,300 \times 1,500 \mu \mathrm{m}^{3}$. The considerably larger field-of-view of this modified microscope design comes at the expense of imaging speed, which is reduced as a result of the lower stiffness of the piezo stages.

It is becoming increasingly clear that to understand how neuronal networks function, it is important to measure neuronal network activity at the system level ${ }^{14,33}$. Our method enables, for the first time, the imaging of activity within the entire CNS of a widely used genetically tractable model organism as it generates multiple fictive behaviours. This work not only opens the door to large-scale circuit analyses in Drosophila in particular but also lays the foundation for a wider set of future studies by providing an integrated strategy for large-scale functional imaging and analysis of neural activity.

\section{Methods}

Genetic constructs and transgenic lines. In live imaging experiments for spatial resolution estimation, a genetic construct in which the histone-2Av gene was fused to the gene encoding enhanced green fluorescent protein was used to fluorescently label the nuclei of all larval cells. In all calcium imaging experiments, the GAL4UAS system ${ }^{48}$ was used to drive expression of the genetically encoded calcium indicator ${ }^{1}$ GCaMP6s. In previous work, a fragment of the promoter for the gene encoding the synaptic protein synaptobrevin was used to create a GAL4 line with panneuronal expression ${ }^{18}$. This GAL4 line (57C10-GAL4) was used to drive expression of GCaMP6s. The LexA-LexOP system ${ }^{49}$ was used to independently drive expression of the red fluorophore tdTomato in anatomically defined regions within the larval CNS. The LexA line (58B03) drove expression in the larval mushroom bodies as well as neuropil regions in the larval CNS.

Animal care and sample preparation. Fly larvae were raised on standard cornmeal-based food. First, second and third instar animals were selected for use in live imaging experiments for spatial resolution estimation. Feeding third instar animals were used for all calcium imaging experiments. In all experiments, individual larvae were dissected in physiological saline containing (in $\mathrm{mM}$ ) $\mathrm{NaCl}$ (135), $\mathrm{KCl}$ (5), $\mathrm{CaCl}_{2}$ (2), $\mathrm{MgCl} 2$ (4), TES (5) and sucrose (36). After being pinned dorsal side up in Sylgard-lined Petri dishes, a dorsal incision was made along the larval body with fine scissors. The body wall was pinned flat and internal organs were removed. The isolated Drosophila CNS was then dissected away and embedded in $1 \%$ low-melting temperature agarose in physiological saline at $36^{\circ} \mathrm{C}$. The agarose containing the CNS was drawn into a glass capillary with $1.4 \mathrm{~mm}$ inner diameter and $2.0 \mathrm{~mm}$ outer diameter, where the agarose quickly cooled to room temperature, forming a soft gel. The agarose cylinder was extruded from the capillary so that the CNS was optically accessible outside of the glass.

Wide-field fluorescence imaging. Using wide-field fluorescence microscopy, activity patterns in isolated nervous systems embedded in agarose and physiological saline were compared with activity patterns in isolated nervous systems exposed only to physiological saline. Preparations were imaged on an Olympus BX51wi upright compound microscope (Olympus, Center Valley, PA) equipped with an OptoLED light source (Cairn Research, Kent, UK) and an Andor DU897 EMCCD camera (Andor Technologies, Belfast, UK). Images were captured at $10 \mathrm{~Hz}$ using Andor IQ software and analysed using custom scripts in Matlab (MathWorks, Natick, MA).

Light-sheet microscope for multi-view functional imaging. We used the conceptual optical arrangement underlying SiMView light-sheet microscopy ${ }^{22}$ as a starting point for designing our high-speed microscope for multi-view functional imaging with one-photon and two-photon excitation (Supplementary Fig. 2). The realization of whole-CNS multi-view functional imaging experiments at up to $5 \mathrm{~Hz}$ (capturing 10 sample volumes per second, with up to 40 images per volume) is the result of four essential advances of the overall design, hardware and software of this earlier microscope, which was capable of multi-view imaging only at up to $0.04 \mathrm{~Hz}$ (capturing 0.16 sample volumes per second, with up to 100 images per volume).

First, and in contrast to single-view functional imaging ${ }^{7}$, multi-view volumetric imaging using piezo positioning of the microscope's detection objectives requires a finely adjustable (micrometre precision) and highly robust (multi-day operation of the imaging system) mechanical solution for relative position and orientation alignment of the detection piezos as well as for optimally matching piezo scan ranges to maximize the microscope's shared multi-view volumetric imaging range We developed a custom two-part mechanical objective positioning system that serves (1) as an adjustable adapter between detection objectives and the piezo stages and (2) as an adjustable base for mounting piezo stages on the optical table (Fig. 1b,c and Supplementary Fig. 2). Technical drawings of these custom parts are provided as Supplementary Data 1. Our custom positioning and rotation adjustment system provides micrometre precision for four key degrees of freedom $x-y$ - $z$-translation of the detection objective and rotation of the piezo base around the normal vector to the optical table. The custom flexures are manufactured from aluminium and designed to minimize mass and maximize stiffness resulting in minimal added load on the piezo stage (thus optimizing speed). The $y$-translation, $z$-translation and rotation degrees of freedom are incorporated in a highly dimensionally stable stainless steel base and are facilitated by low-friction jewelbearing guides. Precise movement is achieved through (1) two differentially adjustable sapphire-tipped micrometres opposing a ruby-tipped spring mechanism for $y$-translation and rotation adjustment, and (2) a micrometre-positioned lowfriction ceramic wedge for $z$-translation. The $x$-translation degree of freedom is incorporated in the flexure using a precision adjustment screw opposing both gravity and a spring preload, and a highly robust one-piece aluminium frame manufactured by wire electrical discharge machining designed to minimize tilt of the optical axis resulting from the objective's weight distribution. Together, these parts allow fine-tuning of the relative position and orientation of the microscope's four objectives with micrometre precision. The ability to perfectly match the limited scan range $(250 \mu \mathrm{m})$ of fast piezo stages with high stiffness is furthermore crucial to maximize the sample volume that can be simultaneously imaged in both detection systems. High-speed imaging with these piezo-stage detection arrangements also requires an imaging chamber design that supports fast synchronized movements of multiple detection objectives while maintaining integrity of the water seal at all objective ports. To this end, we designed a specimen chamber with high-precision custom-moulded silicone rubber seals. These silicone seals are sufficiently thin and flexible to allow unimpeded rapid objective movement across the $250-\mu \mathrm{m}$ piezo travel range while protecting the objective ports from leakage of the physiological saline.

Second, and in contrast to imaging experiments with transparent samples ${ }^{7}$, multi-view functional imaging of a non-transparent sample, with a heterogeneous refractive index distribution that differs significantly from that of the surrounding medium, requires a software framework that accommodates changes in relative position of light sheets and detection objectives as a function of imaging depth into the sample. For this purpose, we introduced a new imaging assay for four-arm functional imaging that allows optimizing microscope alignment on the sample using nonlinear, spatially adaptive, time-dependent operation of all optical arms. This assay enables volumetric multi-view imaging by controlling the piezos that move both detection objectives in synchrony with the galvanometer scanners that move both light sheets. The control software's graphical user interface (GUI) allows the user to define a set of reference planes throughout the sample, which are each 
associated with a unique set of core alignment parameters (specifically, offsets of the two light sheets and positions of the two detection piezos) such that focal shifts resulting from the sample's refractive index distribution and light-sheet refraction resulting from refractive index mismatches at the buffer-to-agarose and agarose-tosample interfaces are mitigated through a locally adaptive microscope alignment. The microscope's electronics framework has furthermore been extended to compensate for the differences in piezo response behaviour in a high-speed imaging setting at the limit of the piezos' operating regime. In this high-speed regime, position-versus-voltage vectors vary from one piezo to the next and are furthermore affected by each unit's individual tuning (for achieving maximum speed while minimizing positional fluctuations and time-consuming over-/ undershoots at the end points of the volumetric scan range). These differences in response behaviour were measured using an oscilloscope, and compensatory adjustments of each unit's voltage wave forms were introduced through the microscope's control framework.

Third, we improved the electronics framework and developed a new microscope control software and image acquisition workflow for piezo-based multi-view imaging, enabling high-speed data acquisition at sustained rates of 370 images per second and data streams of up to 1,000 MB per second. Image data are furthermore streamed in a header-less binary format to a high-performance RAID system. These improvements, together with the changes outlined above, lead to an increase of volumetric imaging speed from 0.8 to $5 \mathrm{~Hz}$, while additionally providing twice as many view angles of the sample. These advances are crucial to match imaging speed to the timescales of activity in Drosophila larval motor circuits and to achieve good physical coverage of the strongly light-scattering Drosophila CNS at the same time.

Fourth, we developed a multi-view functional imaging assay using two-photon excitation, which complements the high-speed imaging mode using one-photon excitation. In this two-photon mode, the microscope offers a maximum volumetric imaging speed of $2 \mathrm{~Hz}$ for the entire Drosophila CNS at high signal-to-noise ratio (Supplementary Movies 3-5), although higher speeds are in principle possible (up to $5 \mathrm{~Hz}$ ) if the respective reduction in signal-to-noise ratio is acceptable. The primary advantages of the two-photon imaging mode are an improvement in signal-to-background ratio and the shift in illumination wavelength from 488 to $940 \mathrm{~nm}$, at which biological tissues generally appear more transparent. In addition, two-photon excitation may be helpful in applications involving concurrent use of genetically encoded calcium indicators and optogenetic effectors.

For detailed information about the components of the hs-SiMView light-sheet microscopy, please see Supplementary Methods.

High-speed volumetric functional imaging. For volumetric calcium imaging of the D. melanogaster larval CNS with light-sheet microscopy, the agarose-embedded larval CNS was transferred to the saline-filled imaging chamber and positioned and oriented using a four-axis mechanical stage that held the specimen stationary in front of the four objectives (Supplementary Fig. 2). The detection arms of the multi-view light-sheet microscope were configured with Nikon $\times 16 / 0.8$ numerical aperture (NA) water immersion objectives with a working distance of $3 \mathrm{~mm}$. The objectives were positioned with PI Hera piezoelectric nano-positioners with a $250-\mu \mathrm{m}$ travel range (Physik Instrumente). For one-photon excitation, the specimen was illuminated with two scanned light sheets at $488 \mathrm{~nm}$, focused by Olympus $\times 4 / 0.28 \mathrm{NA}$ air illumination objectives through custom-designed curved glass windows on the sides of the specimen chamber. At the waist of the light sheet, its thickness was set to $1.5 \mu \mathrm{m}$, and the thickness diverged to $3.0 \mu \mathrm{m}$ in the most remote locations of the field-of-view corresponding to the lateral edges of the specimen (Supplementary Fig. 3). For two-photon excitation, the specimen was illuminated with scanned light sheets at $940 \mathrm{~nm}$, using Nikon $\times 10 / 0.3 \mathrm{NA}$ water-dipping illumination objectives. The light sheets were aligned with the focal planes of the two detection objectives, allowing the two sCMOS cameras to simultaneously obtain images of the illuminated plane of the specimen.

For detailed information about hs-SiMView light-sheet microscope configuration and operation for all high-speed volumetric functional imaging experiments, please see Supplementary Methods.

Spatial resolution analysis. To quantitatively assess spatial resolution as a function of the spatial location within the specimen, we imaged nervous systems of first, second and third instar animals with a ubiquitous nuclear label (w; His2 Avenhanced green fluorescent protein; +), using the same microscope configuration as for imaging the GCaMP6s expressing nervous systems. By labelling all cell nuclei in the animal, the nuclear marker effectively provided blob-like targets suitable for lateral and axial resolution measurements throughout all non-neuropil regions of the CNS, and thus allowed us to estimate spatial resolution in situ under relevant experimental conditions. Volumetric imaging was performed with an axial step size of $2.0 \mu \mathrm{m}$. Three-dimensional spatial resolution maps were then computationally reconstructed from the volumetric images using the automated procedure described in the Supplementary Methods.

Multi-view fusion, processing of calcium imaging data and hs-SiMView data management. We developed a computational pipeline for efficient processing and management of large-scale hs-SiMView image data sets using Matlab (MathWorks, Inc.). All software modules follow a memory-efficient design and take full advantage of multi-core processing architectures, so that typical terabyte-scale hs-SiMView data sets can be rapidly processed on a single computer workstation. By modelling the $3 \mathrm{D}$ geometry of the specimen from raw images, image data were first separated into foreground and background voxels. In order to maximize processing performance throughout the computational framework and reduce data size $>10$-fold, background voxels were discarded and foreground voxels were compressed using our custom block-based lossless compression file format. Multi-view fusion of functional imaging data recorded with the hs-SiMView microscope's two sCMOS cameras was performed by (1) aligning image foreground in multi-view image stacks using a rigid transformation (to correct for physical mismatch of the two cameras ${ }^{22}$ ), and (2) blending the image content by using the geometrical model of the specimen to determine which camera view provided the, respectively, shortest detection path length for each voxel. This procedure ensured that the final image stacks contained the highest-quality image content from each camera view. The image stacks of each time-lapse experiment were subsequently spatially registered to the image stack acquired at the midpoint of the respective time-lapse recording to correct for specimen drift during the recording. 25-percentile (one-photon imaging) and 10-percentile (two-photon imaging) intensity levels were computed in a 70-time point sliding window throughout each time-lapse data set. The resulting reference stacks were used as a baseline for calculating $\Delta F / F$ for every voxel in the time-lapse data set.

All of our software, including source code for high-speed lossless hs-SiMView data compression, multi-view image registration, multi-view image fusion, spatial registration of time-lapse image data and $\Delta F / F$ calculation are provided as Supplementary Data 2 . This archive also contains a user guide with detailed instructions for using the Matlab code.

Wave detection from ROI time series. To extract basic features of segmentally coordinated activity in isolated preparations, we measured changes in fluorescence within $163 \mathrm{D}$ ROIs placed within regions that corresponded to abdominal hemisegments (eight left and eight right). Changes in fluorescence (as $\Delta F / F$ ) were extracted through spatial averaging within each ROI.

Activity typically alternated between periods of minimal responsiveness, and 'bouts' including waves of calcium responses either forward (posterior to anterior) or backward (anterior to posterior). These waves could be identified by examining either movies of activity (Fig. 2 and Supplementary Movies 1-5) or segment traces (for example, Fig. 3a,b). We developed an automated procedure to detect the timing and direction of these waves in a standardized fashion across multiple data sets.

First, the following preprocessing steps were applied to the 16 segment time series. The first $120 \mathrm{~s}$ of data were ignored to avoid response non-stationarities at the beginning of the recording. Each time series was $z$-scored (subtracting the mean and dividing by the s.d.). The average response across all segments was subtracted from each of the individual time series. This step normalizes for the overall increase in activity across all segments that occurs during waves, and instead highlights relative changes across the different segments. Finally, to focus on symmetric patterns, signals from left and right hemisegments were averaged, yielding eight preprocessed time series.

The detection of waves proceeds in two steps. First, we embed the 8D time series in a $2 \mathrm{D}$ space using the singular value decomposition (or PCA). Then, we detect simple features indicative of waves in the resulting $2 \mathrm{D}$ space.

More specifically, given a matrix $\boldsymbol{X}$ that is $8 \times T$, where $T$ is the number of time points, we compute the singular value decomposition as:

$$
\mathbf{U S V}=\mathbf{X},
$$

where $\boldsymbol{U}$ and $\boldsymbol{V}$ are orthogonal matrices containing the left and right singular vectors and $S$ is a diagonal matrix with the singular values. The first two columns of $V, \mathbf{v}_{1}$ and $\mathbf{v}_{2}$, are $T \times 1$ vectors containing a $2 \mathrm{D}$ basis for the data. In the resulting 2D space, we found that waves correspond to rotations away from and back toward the origin; forward and backward waves correspond to rotations in different directions (Fig. 3i,j). Thus, it was natural to use amplitude and phase within this $2 \mathrm{D}$ space as a means for detecting the presence and direction of waves. Specifically, we computed the amplitude and phase as:

$$
\begin{gathered}
\mathbf{r}=\sqrt{\mathbf{v}_{1}^{2}+\mathbf{v}_{2}^{2}} \\
\mathbf{t}=\tan ^{-1}\left(\mathbf{v}_{2}, \mathbf{v}_{1}\right)
\end{gathered}
$$

We detected candidate local peaks in the vector $\mathbf{r}$ (where a local peak is defined as a time point with an amplitude larger than that of its two neighbours, larger than a provided threshold, and separated by at least $3 \mathrm{~s}$ ). For each candidate peak, we computed the slope of the (unwrapped) phase vector $\mathbf{t}$ within a window of $2 \mathrm{~s}$. Forward waves were defined as candidate peaks where the slope was positive and greater than a threshold, and backward waves were defined as peaks where the slope was negative and less than a threshold. There are thus only two primary parameters underlying the procedure: a threshold on amplitude, and a threshold on phase slope. Thresholds of 0.04 and 0.125 were found to produce reasonable results across all data sets, and changing these slightly did not qualitatively affect the detection results.

As a control for detection accuracy, in two data sets, wave times were manually annotated by direct inspection of the segment time series. For each annotated wave, counting the presence of an automatically detected wave (or not) was used to define 
a hit rate. For each automatically detected wave, the absence of an annotated wave (or not) was used to define a false alarm rate. For two data sets, the rates (combined across forward and backward waves) were 93\% hits and 3\% false alarms, and 96\% hits and $8 \%$ false alarms, respectively.

The Matlab source code of our software for automatically detecting and classifying forward and backward waves is included in Supplementary Data 2.

Mapping of whole-CNS activity timing. Several statistical approaches, both exploratory and more targeted, were used to generate computational maps of information flow throughout the CNS, relative to the timing of locomotor waves. In these approaches, statistics are computed on a voxel-by-voxel basis to separately capture the magnitude or informativeness of the response (akin to a measure of signal-to-noise), and the timing of the response in each voxel relative to wave events (detected as described above). Initial surveys of the data were based on simple statistics, such as the s.d. of the response averaged across waves, and the time point of the peak response.

For a more refined analysis, we developed a statistic to measure when and to what extent activity peaks are synchronized with locomotor waves. For each voxel, we create a data set of $N$ time series each of length $T$, triggered around the detection of forward or backward locomotor waves. We then measure how well these activity traces are fit using information about the time relative to the wave detection, in comparison to without this information about time. More specifically, we compare how well the activity traces are fit by a rectangular function of fixed radius $r$ :

$$
f_{\text {time }}\left(t ; \lambda_{0}, \lambda_{1}, \tau\right)=\left\{\begin{array}{cc}
\lambda_{1} & t \in[\tau-r, \tau+r] \\
\lambda_{0} & \text { otherwise }
\end{array},\right.
$$

$\lambda_{0} \leq \lambda_{1}$, to how well they are fit by a flat function:

$$
f_{\text {flat }}(t ; \lambda)=\lambda \text {, }
$$

where $t$ signifies time. We find the optimal parameters for these regression fits according to mean-squared error. Supplementary Fig. 4a shows an example of activity traces for a given voxel and the corresponding fits $f_{\text {time }}$ and $f_{\text {flat }}$.

The optimal fits and their errors can be computed efficiently based on the means and variances of the traces over all waves, and using convolutions. As sample means and variances can be computed efficiently with online updates, this allows these fits to be computed online with minimal memory required.

For the time-based fit, the optimal rectangle location $\tau$ is the peak of the convolution of the mean activity trace with a box filter of length $2 r+1$ :

$$
\tau^{*}=\operatorname{argmax}_{\tau} \frac{1}{2 r+1} \sum_{t \in[\tau-r, \tau+r]} \mu_{t}
$$

where $\mu_{t}$ is the mean activity over all $N$ waves at time $t$. The error of the time-based fit can be computed efficiently as:

$$
\operatorname{err}_{\text {time }}=\frac{1}{T}\left[\sum_{t=1}^{T}\left(\sigma_{t}^{2}+\mu_{t}^{2}\right)-\frac{1}{\left|w\left(\tau^{*}\right)\right|}\left(\sum_{t \in w\left(\tau^{*}\right)} \mu_{t}\right)^{2}-\frac{1}{T-\left|w\left(\tau^{*}\right)\right|}\left(\sum_{t \notin \neq\left(\tau^{*}\right)} \mu_{t}\right)^{2}\right]
$$

where $\sigma_{t}^{2}$ is the sample variance of the traces at time $t$ and $w\left(\tau^{*}\right)=\left[\tau^{*}-r, \tau^{*} r\right]$ is the time interval.

For the time-independent, flat fit, the error is simply the variance:

$$
\operatorname{err}_{\text {flat }}=\frac{1}{T} \sum_{t=1}^{T}\left(\sigma_{t}^{2}+\mu_{t}^{2}\right)-\left(\frac{1}{T} \sum_{t=1}^{T} \mu_{t}\right)^{2}
$$

The Matlab source code of our software for mapping whole-CNS activity timing is included in Supplementary Data 2.

Spatial registration of nervous systems. The Drosophila larval CNS template was constructed following the SyGN ${ }^{30}$ using the open source framework Advanced Normalization Tools (https://github.com/stnava/ANTs). Multiple consecutive time points of GCaMP recordings from six independent CNS preparations were averaged to obtain reference volumes for each data set. The segmental nerves were manually masked to focus the image registration on VNC and brain. During SyGN, the images are first aligned by an affine transform to obtain an initial template.

Both template and individual registrations are then iteratively optimized by a deformable registration, such that the deformation of each individual CNS is minimized ${ }^{30}$. We performed SyGN using B-spline diffeomorphic image registration ${ }^{32}$ with a gradient step of 0.1 and five subsampling factors (in pixels: 10 , $6,4,2$, full sampling) and cross-correlation with a neighbourhood radius of 6 as similarity metric.

Registration quality was assessed using the distance of manually annotated, 3D landmarks in VNC and brain, measured with Image ${ }^{50}$ by two annotators. Landmarks in abdominal segments were annotated using the averaged GCaMP reference volumes. Anatomically corresponding landmarks in the brain lobes, specifically equivalent locations within the mushroom bodies, were annotated using 58B03-LexA,LexOP-tdTomato expression patterns recorded for all specimens (Fig. 6a). The average distance of landmarks in the brain lobes resulted as $16.3 \pm 8.6 \mu \mathrm{m}$ (s.d., $n=60$ ) and the average distance of landmarks in the VNC resulted as $4.5 \pm 5.9 \mu \mathrm{m}$ (s.d., $n=150$ ). We also determined the inter-annotator agreement for the same landmarks and measured average distances of $3.2 \pm 3.0 \mu \mathrm{m}$ (s.d., $n=22$ ) in the brain lobes and $1.4 \pm 1.5 \mu \mathrm{m}$ (s.d., $n=30$ ) in the VNC for the positions labelled by annotators 1 and 2 .

Complementing this distance analysis, we also determined the average overlap of the volumes occupied by each individual CNS in the registered GCaMP reference stacks and the volume occupied by the CNS template, respectively. Volume overlap was measured as the fraction of common foregound voxels in the image stacks after applying an adaptive threshold for foreground segmentation (using a threshold of 0.04 times the mean stack intensity for all data sets). The average volume overlap for the six registered CNS preparations resulted as $95.2 \pm 1.1 \%$ (mean \pm s.d., $n=6$ ).

\section{References}

1. Chen, T. W. et al. Ultrasensitive fluorescent proteins for imaging neuronal activity. Nature 499, 295-300 (2013).

2. Tian, L. et al. Imaging neural activity in worms, flies and mice with improved GCaMP calcium indicators. Nat. Methods 6, 875-881 (2009).

3. Bouabe, H. \& Okkenhaug, K. Gene targeting in mice: a review. Methods Mol. Biol. 1064, 315-336 (2013).

4. Asakawa, K. \& Kawakami, K. Targeted gene expression by the Gal4-UAS system in zebrafish. Dev. Growth Differ. 50, 391-399 (2008).

5. Venken, K. J., Simpson, J. H. \& Bellen, H. J. Genetic manipulation of genes and cells in the nervous system of the fruit fly. Neuron 72, 202-230 (2011).

6. Holekamp, T. F., Turaga, D. \& Holy, T. E. Fast three-dimensional fluorescence imaging of activity in neural populations by objective-coupled planar illumination microscopy. Neuron 57, 661-672 (2008).

7. Ahrens, M. B., Orger, M. B., Robson, D. N., Li, J. M. \& Keller, P. J. Whole-brain functional imaging at cellular resolution using light-sheet microscopy. Nat. Methods 10, 413-420 (2013)

8. Panier, T. et al. Fast functional imaging of multiple brain regions in intact zebrafish larvae using selective plane illumination microscopy. Front. Neural Circuits 7, 65 (2013).

9. Vladimirov, N. et al. Light-sheet functional imaging in fictively behaving zebrafish. Nat. Methods 11, 883-884 (2014).

10. Hammen, G. F., Turaga, D., Holy, T. E. \& Meeks, J. P. Functional organization of glomerular maps in the mouse accessory olfactory bulb. Nat. Neurosci. 17, 953-961 (2014).

11. Prevedel, R. et al. Simultaneous whole-animal 3D imaging of neuronal activity using light-field microscopy. Nat. Methods 11, 727-730 (2014).

12. Grosenick, L., Anderson, T. \& Smith, S. J. Elastic source selection for in vivo imaging of neuronal ensembles. From Nano to Macro, 6th IEEE International Symposium on Biomedical Imaging, 1263-1266 (2009).

13. Bouchard, M. B. et al. Swept confocally-aligned planar excitation (SCAPE) microscopy for high speed volumetric imaging of behaving organisms. Nat. Photon. 9, 113-119 (2015)

14. Alivisatos, A. P. et al. The brain activity map project and the challenge of functional connectomics. Neuron 74, 970-974 (2012).

15. Keller, P. J. \& Ahrens, M. B. Visualizing whole-brain activity and development at the single-cell level using light-sheet microscopy. Neuron 85, 462-483 (2015)

16. Portugues, R., Feierstein, C. E., Engert, F. \& Orger, M. B. Whole-brain activity maps reveal stereotyped, distributed networks for visuomotor behavior. Neuron 81, 1328-1343 (2014).

17. Venken, K. J. \& Bellen, H. J. Genome-wide manipulations of Drosophila melanogaster with transposons, Flp recombinase, and PhiC31 integrase. Methods Mol. Biol. 859, 203-228 (2012).

18. Jenett, A. et al. A GAL4-driver line resource for Drosophila neurobiology. Cell Rep. 2, 991-1001 (2012).

19. Li, H. H. et al. A GAL4 driver resource for developmental and behavioral studies on the larval CNS of Drosophila. Cell Rep. 8, 897-908 (2014).

20. Suster, M. L. \& Bate, M. Embryonic assembly of a central pattern generator without sensory input. Nature 416, 174-178 (2002).

21. Hughes, C. L. \& Thomas, J. B. A sensory feedback circuit coordinates muscle activity in Drosophila. Mol. Cell. Neurosci. 35, 383-396 (2007).

22. Tomer, R., Khairy, K., Amat, F. \& Keller, P. J. Quantitative high-speed imaging of entire developing embryos with simultaneous multiview light-sheet microscopy. Nat. Methods 9, 755-763 (2012).

23. Krzic, U., Gunther, S., Saunders, T. E., Streichan, S. J. \& Hufnagel, L. Multiview light-sheet microscope for rapid in toto imaging. Nat. Methods 9, 730-733 (2012).

24. Huisken, J., Swoger, J., Del Bene, F., Wittbrodt, J. \& Stelzer, E. H. K. Optical sectioning deep inside live embryos by selective plane illumination microscopy. Science 305, 1007-1009 (2004).

25. Keller, P. J., Schmidt, A. D., Wittbrodt, J. \& Stelzer, E. H. K. Reconstruction of zebrafish early embryonic development by scanned light sheet microscopy. Science 322, 1065-1069 (2008).

26. Schmid, B. et al. High-speed panoramic light-sheet microscopy reveals global endodermal cell dynamics. Nat. Commun. 4, 2207 (2013). 
27. Fox, L. E., Soll, D. R. \& Wu, C. F. Coordination and modulation of locomotion pattern generators in Drosophila larvae: effects of altered biogenic amine levels by the tyramine beta hydroxlyase mutation. J. Neurosci. 26, 1486-1498 (2006).

28. Iyengar, B. G., Chou, C. J., Sharma, A. \& Atwood, H. L. Modular neuropile organization in the Drosophila larval brain facilitates identification and mapping of central neurons. J. Comp. Neurol. 499, 583-602 (2006).

29. Avants, B. B. et al. The optimal template effect in hippocampus studies of diseased populations. Neuroimage 49, 2457-2466 (2010).

30. Tustison, N. J. \& Avants, B. B. Explicit B-spline regularization in diffeomorphic image registration. Front Neuroinform 7, 39 (2013).

31. Landgraf, M., Sanchez-Soriano, N., Technau, G. M., Urban, J. \& Prokop, A. Charting the Drosophila neuropile: a strategy for the standardised characterisation of genetically amenable neurites. Dev. Biol. 260, 207-225 (2003).

32. Zlatic, M., Li, F., Strigini, M., Grueber, W. \& Bate, M. Positional cues in the Drosophila nerve cord: semaphorins pattern the dorso-ventral axis. PLoS Biol. 7, e1000135 (2009).

33. Freeman, J. et al. Mapping brain activity at scale with cluster computing. Nat. Methods 11, 941-950 (2014)

34. Cunningham, J. P. \& Yu, B. M. Dimensionality reduction for large-scale neural recordings. Nat. Neurosci. 17, 1500-1509 (2014).

35. Ahrens, M. B. et al. Brain-wide neuronal dynamics during motor adaptation in zebrafish. Nature 485, 471-477 (2012).

36. Bender, J. A., Pollack, A. J. \& Ritzmann, R. E. Neural activity in the central complex of the insect brain is linked to locomotor changes. Curr. Biol. 20, 921-926 (2010)

37. Ridgel, A. L., Alexander, B. E. \& Ritzmann, R. E. Descending control of turning behavior in the cockroach, Blaberus discoidalis. J. Comp. Physiol. A Neuroethol. Sens. Neural Behav. Physiol. 193, 385-402 (2007).

38. Harrison, T. C. \& Murphy, T. H. Motor maps and the cortical control of movement. Curr. Opin. Neurobiol. 24, 88-94 (2014).

39. Mesce, K. A., Esch, T. \& Kristan, Jr. W. B. Cellular substrates of action selection: a cluster of higher-order descending neurons shapes body posture and locomotion. J. Comp. Physiol. A Neuroethol. Sens. Neural Behav. Physiol. 194, 469-481 (2008)

40. Briggman, K. L., Abarbanel, H. D. \& Kristan, Jr. W. B. Optical imaging of neuronal populations during decision-making. Science 307, 896-901 (2005).

41. Ohyama, T. et al. Multilevel multimodal integration enhances behavior selection. Nature 520, 633-639 (2005).

42. Turaga, D. \& Holy, T. E. Image-based calibration of a deformable mirror in wide-field microscopy. Appl. Opt. 49, 2030-2040 (2010).

43. Turaga, D. \& Holy, T. E. Aberrations and their correction in light-sheet microscopy: a low-dimensional parametrization. Biomed. Opt. Express 4, 1654-1661 (2013).

44. Wu, Y. et al. Spatially isotropic four-dimensional imaging with dual-view plane illumination microscopy. Nat. Biotechnol. 31, 1032-1038 (2013).

45. Cao, G. et al. Genetically targeted optical electrophysiology in intact neural circuits. Cell 154, 904-913 (2013)

46. Ting, C. Y. et al. Focusing transgene expression in Drosophila by coupling Gal4 with a novel split-LexA expression system. Genetics 188, 229-233 (2011).
47. Potter, C. J., Tasic, B., Russler, E. V., Liang, L. \& Luo, L. The Q system: a repressible binary system for transgene expression, lineage tracing, and mosaic analysis. Cell 141, 536-548 (2010).

48. Brand, A. H. \& Perrimon, N. Targeted gene expression as a means of altering cell fates and generating dominant phenotypes. Development 118, 401-415 (1993).

49. Lai, S. L. \& Lee, T. Genetic mosaic with dual binary transcriptional systems in Drosophila. Nat. Neurosci. 9, 703-709 (2006).

50. Schneider, C. A., Rasband, W. S. \& Eliceiri, K. W. NIH Image to ImageJ: 25 years of image analysis. Nat. Methods 9, 671-675 (2012).

51. Mellert, D. J. \& Truman, J. W. Transvection is common throughout the Drosophila genome. Genetics 191, 1129-1141 (2012).

\section{Acknowledgements}

We thank Brian Coop and the Janelia ID\&F team for mechanical designs and custom mechanical parts; Michael Coleman for custom microscope operating software; Misha Ahrens, Marta Zlatic, Vivek Jayaraman and Allan Wong for their helpful discussions and valuable comments on the manuscript; Jim Truman, Gerry Rubin, Karen Hibbard and the Janelia Fly Facility for sharing and maintaining transgenic Drosophila stocks; Tim Bayley for assistance in developing the larval CNS imaging preparation, and William Rowell (Janelia Technical Resources Group) for help with processing Olympus BX51wi image data. This work was supported by the Howard Hughes Medical Institute.

\section{Author contributions}

W.C.L., S.R.P. and P.J.K. designed experiments. P.J.K., W.C.L. and K.M. designed and built the hs-SiMView microscope. W.C.L. and S.R.P. conducted experiments. J.F., K.B., B.H. and P.J.K. developed data analysis methods. W.C.L., S.R.P., B.H., K.B., J.F. and P.J.K. analysed data. P.J.K. and S.R.P. wrote the manuscript with input from all authors.

\section{Additional information}

Supplementary Information accompanies this paper at http://www.nature.com/ naturecommunications

Competing financial interests: The authors declare no competing financial interests.

Reprints and permission information is available online at http://npg.nature.com/ reprintsandpermissions/

How to cite this article: Lemon, W. C. et al. Whole-central nervous system functional imaging in larval Drosophila. Nat. Commun. 6:7924 doi: 10.1038/ncomms8924 (2015).

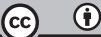

This work is licensed under a Creative Commons Attribution 4.0 International License. The images or other third party material in this article are included in the article's Creative Commons license, unless indicated otherwise in the credit line; if the material is not included under the Creative Commons license, users will need to obtain permission from the license holder to reproduce the material To view a copy of this license, visit http://creativecommons.org/licenses/by/4.0/ 